\title{
SIMULATIONS OF BLUFF BODY FLOW INTERACTION FOR NOISE SOURCE MODELING
}

\author{
Mehdi R. Khorrami", David P. Lockard ${ }^{* *}$, Meelan M. Choudhari ${ }^{*}$, Luther N. Jenkins***, \\ Dan H. Neuhart ${ }^{* * *}$, and Catherine B. McGinley ${ }^{* * *}$ \\ NASA Langley Research Center \\ Hampton, VA
}

\begin{abstract}
The current study is a continuation of our effort to characterize the details of flow interaction between two cylinders in a tandem configuration. This configuration is viewed to possess many of the pertinent flow features of the highly interactive unsteady flow field associated with the main landing gear of large civil transports. The present effort extends our previous two-dimensional, unsteady, Reynolds Averaged Navier-Stokes computations to three dimensions using a quasilaminar, zonal approach, in conjunction with a two-equation turbulence model. Two distinct separation length-to-diameter ratios of $L / D=3.7$ and 1.435 , representing intermediate and short separation distances between the two cylinders, are simulated. The Mach 0.166 simulations are performed at a Reynolds number of $\operatorname{Re}=1.66 \times 10^{5}$ to match the companion experiments at NASA Langley Research Center. Extensive comparisons with the measured steady and unsteady surface pressure and off-surface particle image velocimetry data show encouraging agreement. Both prominent and some of the more subtle trends in the mean and fluctuating flow fields are correctly predicted. Both computations and the measured data reveal a more robust and energetic shedding process at $L / D=3.7$ in comparison with the weaker shedding in the shorter separation case of $L / D$ $=1.435$. The vortex shedding frequency based on the computed surface pressure spectra is in reasonable agreement with the measured Strouhal frequency.
\end{abstract}

\section{Introduction}

Prediction and modeling of noise sources associated with a landing gear, in particular the main gear, is one of the more challenging tasks facing today's airframe noise modelers. To understand the noise generation mechanisms requires an in-depth knowledge of the local, unsteady flow field. Given the

\footnotetext{
*Aerospace Technologist, Computational AeroSciences Branch, Associate Fellow AIAA

** Aerospace Technologist, Computational AeroSciences Branch, Senior member AIAA

*** Aerospace Technologist, Flow Physics and Control Branch
} 
geometric complexity of a main landing gear, sufficiently resolving the highly interactive and nonlinear time-dependent flow regime for physics-based noise modeling is a daunting task. A more prudent approach is to break down the main gear geometry into simpler subcomponents that allow a more detailed study of the flow field. A canonical configuration that models a variety of component level interactions is that of multiple cylinders in a tandem arrangement. The present effort is directed at the case of two identical cylinders spatially separated in the streamwise direction (Fig.1).

Tandem cylinder flow field has received considerable attention in that past with regard to such diverse areas as heat exchanger fins, chimney stacks, and off-shore oil platforms, to name a few. However, the majority of the past studies were restricted to the low Reynolds (Re) number flow regime that is dominated by laminar flow separation and periodic vortex shedding. Detailed review of the previous investigations and an overview of the global features of the tandem cylinder flow are provided in references 1-3 and therefore, will not be repeated here. It suffices to point out that, according to references 1-3, three distinct flow regimes are dominant. They correspond to short, intermediate, and large separation distances between the cylinders' centerlines. In the case of short separations, the two cylinders behave as a single bluff body with vortex shedding occurring at the rear cylinder only. For large separation distances, the flow field approaches that of isolated single cylinders. At intermediate separation distances, the flow regime is "critical", whereby, it can switch between bi-stable flow states representing short and large separation distances. Measurements of various flow quantities, highlighting different aspects of the tandem cylinder flow field, are reported in references 4-15. Numerical simulations at fairly low Reynolds numbers are considered in references 16-18.

Despite their usefulness, the earlier studies lack detailed on-surface and off-surface measurements that are needed for fully characterizing the turbulent fluctuating field and benchmarking the computations. The present computational work is part of a larger multi prong study, comprising of both experiments and computations that is aimed at characterizing the complex interactive flow field around tandem cylinders at a relatively high Reynolds number. The experiments were conducted in several phases in the Basic Aerodynamic Research Tunnel (BART) at NASA Langley Research Center (LaRC). Phase 1 of the experiment, discussed by Jenkins et al. ${ }^{19}$, was targeted at providing steady surface pressures and detailed off-surface measurements of the flow field using Particle Image Velocimetry (PIV). In addition, hot-wire measurements in the wake of the rear cylinder were also conducted. Phase 2 of the experiment mainly focused on collecting steady and unsteady surface pressure data. A detailed account of phase 2 measurements is provided by Jenkins et al. ${ }^{20}$. 
Phase 1 experiments were conducted at a Reynolds number of $\mathrm{Re}=1.66 \times 10^{5}$ based on the cylinder diameter and the maximum tunnel speed of $M=0.166$. Phase 2 experiments employed larger cylinders to allow more instrumentation on the cylinders. To maintain the Reynolds number, the flow speed was decreased to $M=0.129$. To ensure a fully turbulent shedding process, the boundary layers on the front cylinder were tripped between azimuthal locations of 50 and 60 degrees from the leading stagnation point. The measured surface pressure distribution for the large separation case was nearly identical to that measured by previous investigators for a single, isolated cylinder at a Reynolds number greater than 8 million.

Exploratory noise measurements, documenting radiated sound field for various cylinder arrangements, were performed by Hutcheson and Brooks ${ }^{21}$ in the Quiet Flow Facility at LaRC. This initial noise data will be supplemented by more extensive acoustic measurements (focused only on tandem orientation) in the near future.

The parallel computational effort was also executed in multiple stages. Our initial study, discussed by Khorrami et al. ${ }^{22}$, was restricted to two-dimensional (2-D) fully turbulent simulations using standard unsteady Reynolds averaged Navier-Stokes (URANS) equations in conjunction with Menter's ${ }^{23}$ twoequation turbulence model. Separation distances of $\mathrm{L} / \mathrm{D}=1.435$ and 3.7 were considered. Minor modifications to the 2-D grids of ref. 22 provided slight improvements in the computed URANS solutions. An account of the latter 2-D results can be found in Khorrami, Choudhari, Lockard, Jenkins, and $\mathrm{McGinley}^{24}$ (hereafter referred to as KCLJM). The 2-D results from ref. 22 and KCLJM, while showing reasonable agreement with the measured steady surface pressure, failed to match some of the prominent features of the off-surface mean and fluctuating velocity fields. Apart from the shortcomings associated with the 2-D nature of the simulations, those computations proved to be overly diffusive due to the effects of turbulence model. In fact, for $\mathrm{L} / \mathrm{D}=1.435$, the simulations failed to produce any meaningful flow unsteadiness and the computed solutions reverted to a steady state. On the positive side, the 2-D URANS results were instrumental in illustrating the extreme sensitivities of the tandem cylinder configuration (particularly at short L/Ds) to small inflow angularities.

The present work extends our previous 2-D simulations to 3-D in order to eliminate some of the past shortcomings and to document three-dimensional effects on the dynamics of the flow field. To remedy the excessive diffusiveness of the turbulence model, a quasi-laminar zonal approach is advocated and shown to produce very encouraging results. Data from phase 1 and phase 2 measurements are used to provide a direct comparison with the present simulations. 


\section{Computational Approach}

The overall computational procedure is similar to our earlier 2-D simulations described in KCLJM. Only the pertinent issues relevant to the current 3-D simulations are presented.

\subsection{Flow Solver}

As in our earlier effort, the CFL3D flow solver is used to compute the flow field. Briefly, the CFL3D code is a finite-volume flow solver based on the compressible form of 3-D, time-dependent, thinlayer Navier-Stokes (N-S) equations. Provisions for solving the full N-S equations have been added to the code recently. However, preliminary results (for the present configuration) based on the full N-S equations revealed $20 \%$ to $30 \%$ increase in the computational cost relative to the thin layer approximation, without any noticeable improvement in the computed solution. Therefore, all the simulated results presented in this paper are based on the thin-layer N-S approximation.

All current computations are performed using the second-order-accurate time discretization and the "dual time-stepping" $\tau$-TS method. ${ }^{25}$ Seventeen to thirty subiterations, in conjunction with 2- or 3-level V-type multi-grid cycles, are employed to achieve two orders of magnitude drop in residuals during each time step.

\subsection{Turbulence Model and Zonal Approach}

For the standard URANS computations, the 2-equation Shear Stress Transport (SST) model of Menter $^{23}$ is used. Due to the overly diffusive nature of the turbulence model, the initial URANS computation revealed no sign of developing 3-D structures within the flow field, despite transient forcing with a broadband spanwise spectrum. In fact, except for a low frequency and low amplitude spanwise undulation of the shed vortices, the flow field essentially remained 2-D. To remedy this shortcoming, given the previous success of our zonal approach ${ }^{26}$, the flow field is assumed to be quasi-laminar, except for a narrow strip surrounding the cylinder surfaces. In this approach, the flow interior to the strip is treated as fully turbulent using the SST turbulence model. Outside of this strip, the production term associated with the turbulence model is switched off. In figure 2 the extent of the fully turbulent flow zone surrounding each cylinder is highlighted with the purple color grid. The radial extent of the strip is not constant due to the slight azimuthal non-uniformity of the grid stretching. The minimum and maximum thicknesses of the strip are on the order of 0.01D and 0.04D respectively. Presently, we do not have a rule of thumb on how to arrive at an optimal width for the turbulent zone. The current width was selected based on our past experiences with similar issues involving high-lift flow computations and a 
desire to keep the pre-separated boundary layers on the cylinders immersed within the fully turbulent zone.

\section{3 $\underline{\text { Simulated Configurations and Corresponding Grids }}$}

The simulated tandem cylinder configuration is comprised of two cylinders of equal diameter (D) aligned along the streamwise direction. The geometry under consideration and the computational coordinate axes (fixed to the leading edge of the front cylinder) are shown in Fig. 1. The diameter of the cylinders employed during the phase 1 and phase 2 experiments corresponds to $\mathrm{D}=0.04445 \mathrm{~m}(1.75$ inches) and $0.0515 \mathrm{~m}$ (2.25 inches), respectively. The cylinders span the entire tunnel height of $\mathrm{b}=0.71 \mathrm{~m}$ (28 inches), such that the aspect ratio during the two experiments equals $\mathrm{b} / \mathrm{D}=16$ and 12.4 , respectively. In accordance with our previous 2-D computations (KCLJM), two distinct separation distances of $\mathrm{L} / \mathrm{D}=$ 1.435 and 3.7 are considered during the 3-D simulations.

The x-y planar grid and mesh distribution were presented in KCLJM and the reader is referred to that paper for more detailed information. It suffices to mention that the block structured, $x-y$ planar grid contains a total of $404 \mathrm{~K}$ and $318 \mathrm{~K}$ points for $\mathrm{L} / \mathrm{D}=3.7$ and 1.435 , respectively. Care was taken so that the first point off the solid surfaces is at $y^{+}<1$. A spanwise extent, Lz, of three diameters was selected for constructing the 3-D domains. The three diameters width was deemed to be a good compromise between balancing the computational cost and capturing the prominent 3-D flow features. To generate the 3-D grids, the 2-D grids were replicated along the span at 97 equally spaced points (Fig. 3). Periodic boundary conditions are employed in the spanwise direction. The total grid count for $\mathrm{L} / \mathrm{D}=3.7$ and 1.435 are 40 million and 31 million points respectively, representing our finest resolution. Similar to KCLJM, simulations based on medium-level grids (derived from the finest grid by removing every other grid points in each direction) were performed to allow an assessment of the grid convergence of the computed solutions.

During the course of the current study, the adequacy of the selected three-diameter span became questionable. To test the effect of a larger span on the time-dependent flow field, a new grid with $\mathrm{Lz}=6$ (i.e., twice the original span) was generated for $\mathrm{L} / \mathrm{D}=3.7$ separation. The new grid was constructed using the medium $x-y$ planar mesh but maintaining our fine resolution along the span, resulting in 193 spanwise points for a total grid count of 20 million. 


\section{Results}

For ease of comparison with the companion experiments, and interpretation of the relevant trends, the computed results are processed and presented in a non-dimensional fashion. The nondimensionalization is with respect to the cylinder diameter D and the free-stream speed, density, and molecular viscosity. For the present case, the reference flow variables were set to match the conditions at the entrance to the BART test section during the phase 1 experiment when PIV measurements were obtained. The flow conditions for the simulations correspond to $M=0.166$ and $\operatorname{Re}=1.66 \times 10^{5}$. Given the negligible compressibility effects at such low Mach numbers, the current simulations are also applicable to the phase 2 measurements, which were conducted with larger cylinders at the same Re but a lower M. Due to the negligible effects of small inflow angularity on the flow field for intermediate and large separations, the $\mathrm{L} / \mathrm{D}=3.7$ simulations are conducted with an angle of attack (AoA) of 0 degrees. However, to better replicate the flow asymmetry of shorter separations as reported in KCLJM, simulations involving the $\mathrm{L} / \mathrm{D}=1.435$ configuration are performed with an AoA of 1 degree.

A constant non-dimensional time step of $\Delta t=0.01693$ (corresponding to 150 points per period for a $500 \mathrm{~Hz}$ signal) is used for all simulations. The simulations are run first in a steady-state mode, and then in unsteady manner, with random suction and blowing applied to different spanwise and azimuthal sections of both cylinder surfaces. The forcing did not exceed $3 \%$ of the freestream velocity. The forcing was turned off after shedding was observed, typically 200 time steps into the unsteady calculation. The simulations were then run for 5000-10,000 time steps to allow the transient flow field to wash out before collecting time records. Averaged flow quantities are produced by time-averaging over 10,000-15,000 samples followed by spatial-averaging over the span. In addition, to allow direct comparison with the PIV measurements, the 2-D planar definition of turbulent kinetic energy (TKE) involving the $\mathrm{x}-\mathrm{y}$ components of velocity fluctuations is used through out this paper.

The $\mathrm{L} / \mathrm{D}=3.7$ case has been the primary focus of attention during the analysis of the simulation database. As will be seen in the following sections, the flow field at this L/D is post-critical, i.e., the tandem cylinders behave as independent bluff bodies with a robust vortex shedding process established in the wake of each cylinder. For $\mathrm{L} / \mathrm{D}=1.435$, unlike the fully turbulent URANS computations of KCLJM, the current quasi-laminar zonal simulations produce and maintain a fully unsteady flow field. However, the 1.435 arrangement turns out to be a more difficult case to simulate. This is mainly due to the extreme sensitivity of the flow to the inflow angularity and the resulting low frequency flow dynamics within the gap region. Determining an appropriate value of the inflow AoA to match with the experiment has proven 
difficult. The results indicate that an AoA of less than 1 degree might have been more appropriate to match the combined on- and off-surface flow fields measured in the experiment.

\subsection{Mean and Fluctuating Surface Pressure Fields}

The surface pressure distributions, $\mathrm{Cp}$, for the $\mathrm{L} / \mathrm{D}=3.7$ separation are shown in Fig. 4. For the sake of completeness, the fully turbulent, 2-D, URANS results of KCLJM are also included. Recall from Fig. 1 that the azimuthal angle $\theta$ is zero in the upstream direction and increases in the clockwise direction. On the front cylinder, the current simulations capture the relevant trends and produce $\mathrm{Cp}$ values that are close to the measured ones (Fig. 4a). The 3-D fine grid and the large span solutions slightly under-predict the peak suction and its distance from the front stagnation, but properly capture the boundary layer separation locations. The larger-span result shows a modestly better pressure recovery at the base of the cylinder. On the rear cylinder (Fig. 4b), the front stagnation pressures are correctly captured. Similar to the front cylinder, however, the peak suction and its location are substantially under-predicted. The CFD results show an earlier boundary layer separation which indicates that a wider wake is being computed. Notice that neither the finer grid nor the doubling of the span improved the rear cylinder pressure distribution suggesting the turbulence model to be the primary factor.

The root-mean-square (rms) pressure fluctuations on the solid surfaces are shown in Fig. 5. The location of the peak fluctuations on the front cylinder, occurring close to the separation point, is accurately predicted. Note that 3-D effects significantly reduce the magnitude of the pressure fluctuations over the entire surface bringing the values closer to the measurement. Doubling the computational span further reduces the computed rms values producing remarkably good agreement with the measured data. In agreement with previous measurements ${ }^{11-12}$, the rear cylinder displays much higher rms pressure amplitudes relative to the front cylinder. On the rear cylinder, the peaks occur at $\theta=40$ and 320 degrees, corresponding to the locations where the shed vortices from the upstream cylinder impact the surface of the downstream one. The secondary peaks at $\theta=95$ and 265 degrees are associated with the boundary layer separation on the rear cylinder. Except for the peak magnitude, the larger span solution tends to provide a better agreement with the measurements. Surprisingly, the best agreement at the base of the rear cylinder is provided by the 2-D URANS solution.

The surface pressure spectra at $\theta=135$ degrees are plotted versus Strouhal (St) frequency in Fig. 6 for $\mathrm{L} / \mathrm{D}=3.7$. As expected, the measured spectra show a prominent tonal behavior, reinforcing the notion that each cylinder acts as an independent body. The essence and character of the pressure spectra are reasonably well predicted across the entire frequency range. Generally speaking, the fine grid solution 
better captures the shape of the measured spectra (in particular at higher frequencies) while the larger span solution produces better agreement with regard to the amplitude. For the measured spectra, vortex shedding occurs at $\mathrm{St}=0.242$ for both front and rear cylinders. The corresponding shedding frequencies extracted from the medium grid, fine grid, and larger span spectra are 0.235, 0.207, and 0.215, respectively.

Effective modeling of the noise sources depends on acquiring a good knowledge of the source characteristics and distribution along the span direction. Spanwise correlations ${ }^{27}$ based on the surface pressure fluctuations at $\theta=135$ degrees are plotted in Fig. 7 for $\mathrm{L} / \mathrm{D}=3.7$. The experimental data, showing some scatter, represents all possible spanwise separations between different pairs of surface mounted transducers. Showing a behavior that is typical of a single cylinder in fully turbulent flow, the pressure field remains correlated up to 5-6 diameters. Surprisingly, our earlier hot-wire measurements ${ }^{20}$ in the intermediate wake of the rear cylinder revealed a much faster decay in the lateral correlation that was on the order of one diameter. The correlations from the simulations were performed in the time domain using the entire record of the surface pressure. The computed correlations, based on the fine grid and larger span solutions, capture the initial decay but fail to predict larger separations due to insufficient width of the computational domain. Similar to the measured data, the computed correlations also contain certain level of scatter. A sample scatter plot for the correlation on the front cylinder is shown in Fig. 7c. We anticipate the scatter levels would diminish significantly if longer time records were obtained.

The post-processed surface pressure results for $\mathrm{L} / \mathrm{D}=1.435$ are displayed in Figs. 8-11. We have not yet had the chance to evaluate the effects of a larger spanwise domain on the $\mathrm{L} / \mathrm{D}=1.435$ flow field. The predicted mean $\mathrm{Cp}$ distributions on the front cylinder for various grids (Fig. 8a) are in good agreement with the measured profile. The pressure distribution on the rear cylinder (Fig. $8 \mathrm{~b}$ ) shows a noticeable asymmetry potentially caused by the entrance flow angularity in the BART facility as discussed in reference 19. The simulated solutions, all obtained with the free-stream AoA $=1$ degree, mimic the asymmetric character of the measured pressure distribution. However, the agreement between computed and measured surface pressures on the rear cylinder is less satisfactory than that on the front cylinder. For this $\mathrm{L} / \mathrm{D}$, the medium grid solution displays a peculiar behavior in the pressure recovery region near the base of the rear cylinder resulting in a local suction peak. The pressure recovery shows a marked improvement with increasing resolution. The rms pressure fluctuations are plotted in Fig. 9. The inadequacy of the medium grid in capturing the correct rms profile is clearly evident from the figure. The coarser grid over-predicts the rms pressures by a wide margin and, in the case of the rear cylinder, produces a profile that shows little resemblance to the measured profile. The better resolved solution brings the rms amplitudes down to levels that are more in line with the experimental values. Nevertheless, 
the fine grid rms profiles show a degree of asymmetry that is not fully supported by the measurements. In hindsight, as alluded to earlier, it seems that our selected inflow AoA of 1 degree may have been too high. In future, we may repeat the $\mathrm{L} / \mathrm{D}=1.435$ simulations at a smaller AoA.

The measured and computed surface pressure spectra at $\theta=135$ degrees are shown in Fig. 10. In agreement with the PIV results of Jenkins et al. ${ }^{19}$, the measured spectra depict a more benign flow field, lacking dominant tonal shedding, with pressure fluctuations that are substantially lower in amplitude relative to the $\mathrm{L} / \mathrm{D}=3.7$ configuration. For the front cylinder, the measured spectrum shows a minor peak at $\mathrm{St}=0.19$ indicating a weak shedding process. Rather than showing a tonal peak, the rear-cylinder spectrum possesses a low-amplitude, very broad hump that is centered at $\mathrm{St}=0.243$. In contrast, the computed spectra for both cylinders display a very robust tonal behavior with shedding frequencies of 0.19 and 0.208 for medium and fine grids, respectively. The coarser grid spectrum shows significantly higher fluctuation energy at low and medium frequencies. Increased resolution lowers the pressure levels considerably, and, in the case of the rear cylinder (Fig. 10b), produces a spectrum shape that closely follows the measured profile.

The spanwise correlation, based on surface pressure fluctuations at $\theta=135$ degrees, are plotted in Fig. 11. Both the measurements and fine grid simulation reveal a much smaller correlation length scale than that observed for the $\mathrm{L} / \mathrm{D}=3.7$ arrangement. The better resolved computation over-predicts the initial correlation decay for the front cylinder and under-predicts it for the rear one. Nevertheless, the computed result is not far off the mark and viewed to be reasonable. Despite the rapid decay of the lateral correlations shown in Fig. 11, the issue of performing the simulation with a larger spanwise domain needs to be revisited in the future.

\subsection{Off-Surface Flow Field: Qualitative Comparison}

In this section an overall view of the tandem cylinder flow field is provided by comparing the simulated results with the PIV measurements of Jenkins et al. ${ }^{19}$. For $\mathrm{L} / \mathrm{D}=3.7$, the predicted velocity field from the three grids considered is in remarkably close agreement with the experimental data. For this cylinder separation, a general observed trend is that the PIV measurements typically fall in between the results obtained from the fine grid solution and those extracted from the larger span simulation. Therefore, due to the limited available space, we restrict our presentation of the post-processed results to the larger span solution for $\mathrm{L} / \mathrm{D}=3.7$ case and the fine grid solution for the 1.435 separation.

Computed mean flow field contours for $\mathrm{L} / \mathrm{D}=3.7$ are plotted in Fig. 12. It is evident that all prominent features of the gap flow are captured. Notice that the front cylinder wake only partially 
recovers before reaching the rear cylinder. In contrast to the 2-D URANS results of KCLJM, the mean vortex roll-up location between the two cylinders, as depicted by the regions of high vertical velocities, is in good agreement with PIV measurements. A more stringent test to evaluate the fidelity of the simulated results involves the comparison of the fluctuating velocity fields. Contours of the streamwise and vertical fluctuation intensities and the $\mathrm{x}-\mathrm{y}$ planar TKE are shown in Fig. 13. Notice that the primary features, as well as some of the more subtle features of the turbulent fluctuating field, are well resolved and in good agreement with the measurements. Note that for this intermediate separation distance, the immediate wake of the front cylinder is devoid of TKE (Fig. 13e). The primary turbulent hotspot resides in the middle of the gap region and is mostly associated with the vortex roll-up process. A secondary region of elevated TKE activity is confined to the leading edge of the rear cylinder. Figure $13 \mathrm{c}$ clearly demonstrates that the vertical velocity fluctuations are responsible for this elevation at the leading edge. A byproduct of these excess fluctuations would be a non-stationary front stagnation point which will manifest itself as lift oscillations.

The corresponding flow contours for the $\mathrm{L} / \mathrm{D}=1.435$ separation are shown in Fig. 14. Overall, the agreement with the measured field is reasonable. Notice the PIV measurement displays an asymmetric flow (Fig. 14d) within the gap region between the two cylinders, highlighting the likely sensitivity to a skewed background flow. The computed mean vertical velocity (Fig. 14c), obtained with AoA = 1 degree, shows a strong positive (upward) component on the front surface of the rear cylinder consistent with computed prediction based on a nonzero flow angularity. However, the predicted magnitude of the upwash inside the gap region is higher than that observed in the experiment. This over-prediction can be traced to two somewhat independent factors. One contributing factor involves the selected AoA value for the current computations which, as indicated in the previous section, may have been too high. An equally important factor relates to the establishment of a weak spanwise flow within the gap area due to finite span model and tunnel sidewall effects in the experiment. While the AoA effects can be simulated numerically, the present spanwise periodic flow setup is incapable of simulating tunnel installation effects.

The computed mean spanwise vorticity field (Fig. 14e) shows relatively good agreement with the measured field except for a region of excess positive vorticity on the lower front surface (between $\theta=290$ and 0 degrees) of the rear cylinder. Scrutiny of the instantaneous flow field revealed that at the reattachment point, the shear layer vortices (produced by the amplifying Kelvin-Helmholtz instabilities) mostly take an upward path through the gap that is adjacent to the rear cylinder surface rather than moving in the streamwise direction. The path alteration is caused by the forward movement of the bottom reattachment point due to the enforced flow angularity. The planar TKE, in the wake of the rear cylinder, 
is plotted in Fig. 14g. Note the predicted energy levels are much higher than the corresponding measurement. In addition, the figure shows a significant asymmetry in the computed TKE field that is not supported by the PIV data.

\subsection{Off-Surface Flow Field: Quantitative Comparison}

The previous section provided a global view of the computed flows. To highlight the merits and shortcomings of the present zonal approach in capturing a complex, fully turbulent, interactive flow field, we resort to a more direct quantitative comparison. Due to space limitations, only sample coordinate cuts along certain $\mathrm{x}$ and $\mathrm{y}$ locations are presented. The locations of these cuts were selected to maximize the level of flow-field information conveyed to the reader. For $\mathrm{L} / \mathrm{D}=3.7$ case, profiles along the vertical cuts at $\mathrm{X}=1.5 \mathrm{D}$ downstream of the front cylinder center and at $\mathrm{X}=0.75 \mathrm{D}$ downstream of the rear cylinder center are presented. The horizontal (centerline) cuts are along the geometric symmetry line and comprise both the gap region and wake of the rear cylinder. The x-profiles are presented in the computational coordinates that are fixed at the leading edge of the front cylinder. For $L / D=1.435$, only $x-y$ cuts downstream of the rear cylinder are shown.

The mean velocities and TKE profiles downstream of the front cylinder for the L/D $=3.7$ arrangement are displayed in Fig. 15. The extracted profiles, representing the near wake region, are in very good agreement with the measurements. Note that the larger span solution shows a deeper wake (caused by the downstream shift in the roll-up location relative to the other two solutions). Both the energy levels and spatial distribution of the fluctuating field are properly captured and predicted. The corresponding near wake profiles, downstream of the rear cylinder, are shown in Fig. 16. Once again, the agreement between experiment and computations is quite encouraging. Figure 17 presents horizontal profiles of mean streamwise velocity and TKE in the gap region for $\mathrm{L} / \mathrm{D}=3.7$. The larger span solution over-predicts the roll-up (maximum velocity deficit) location and under-predicts the gap-flow recovery as the wake approaches the rear cylinder (Fig. 17a). However, this solution provides near perfect agreement for the centerline TKE distribution (Fig. 17b). The fine grid simulation slightly under-predicts the roll-up location and produces a more energetic near-wake fluctuating field. Obviously, it would be quite informative to repeat the wider span simulation using the finer $x-y$ planar grid. The mean streamwise velocity and TKE distribution along the centerline downstream of the rear cylinder are displayed in Fig. 18. The computed mean velocity profiles (Fig. 18a) fall within a narrow band of each other and all three of them predict a longer recirculation zone and a larger wake deficit. Both of these wake attributes can be related to the lack of model installation (3-D) effects that are present in the experiment. The simulated TKE profiles capture the pertinent features of the rear wake fluctuation field. The larger span solution 
provides the best fit with regard to the TKE maximum levels and streamwise location of the peak fluctuations. However, the predicted curves show a slower decay in the downstream direction.

The extracted profiles for the $\mathrm{L} / \mathrm{D}=1.435$ separation are presented in Fig. 19 and Fig. 20. Both figures reveal rather clearly the shortcomings of the medium grid solution in capturing the relevant flow features associated with the smaller gap arrangement. Increasing the resolution markedly improves the quality of the solution and provides reasonable agreement with the measured profiles. Figures $19 \mathrm{c}$ and $20 \mathrm{~b}$ show the wake fluctuations to be less energetic and peak farther downstream than the corresponding rear cylinder wake for $\mathrm{L} / \mathrm{D}=3.7$ (Figs. 16c and 18b). The combination of these two effects explains the lack of a visible shedding footprint in the surface pressure spectrum shown in Fig. 10b. While the fine grid solution captures the prominent features of the TKE field, it consistently over-predicts the levels. Reiterating our earlier comments, we think performing the computation for the $\mathrm{L} / \mathrm{D}=1.435$ arrangement with a smaller AoA may partially alleviate some of the observed differences with the measurements.

\subsection{Instantaneous Flow Field}

Instantaneous iso-surfaces of vorticity for $\mathrm{L} / \mathrm{D}=3.7$ are presented in Fig. 21 . The figure shows that soon after separating from the front cylinder, the shear layer amplifies the Kelvin-Helmholtz instabilities which manifest themselves as spanwise rollers (Fig. 21a). Due to onset of secondary instabilities, rapid breakdown of the spanwise rollers ensues resulting in 3-D vorticity lumps and partial realignment of the vorticity field into streamwise vortices (Fig. 21b). Surprisingly, at this L/D, only a minute fraction of the spanwise vorticity is converted into the vertical component. A sample snapshot of the simulated vorticity field at a constant $\mathrm{z}$ plane is shown in Fig. 22. The plot which closely mimics the PIV snapshot, shows the roll-up process as an amalgamation of the smaller size rollers into a large structure that resembles a typical shed vortex.

The corresponding iso-surface of vorticity fields for $\mathrm{L} / \mathrm{D}=1.435$ are shown in Fig. 23. For this gap distance, the 3-D breakdown of the spanwise rollers is underway when the shear layer impacts and reattaches to the rear cylinder (Fig. 23a). In contrast to the flow field for $\mathrm{L} / \mathrm{D}=3.7$, a significant portion of the initial vorticity field is converted into the vertical vorticity component (Fig. 23b).

\section{Conclusion}

Simulations of tandem cylinders have highlighted the sensitivity of the results to the grid resolution and turbulence model. Detailed comparisons with experimental data has shown that important flow features for noise source modeling can be missed unless extreme care is taken to capture all of the 
relevant flow phenomena. Simple comparisons of the surface pressure distribution and lift spectra are important but insufficient to assess the overall adequacy of the solution. The extensive experimental results used in this work allowed us to identify several deficiencies in our calculations. Our zonal turbulence model approach is a clear improvement over standard URANS, but the ad-hoc nature of the extent of the RANS zone is one of several deficiencies that need to be improved. For larger cylinder separation distances, the flow is correlated over a relatively large portion of the span requiring the simulations to be much larger than was originally anticipated. Doubling the length of the span clearly improved the solution for $\mathrm{L} / \mathrm{D}=3.7$, but further study is needed for the $\mathrm{L} / \mathrm{D}=1.435$ case. Furthermore, the solutions for both separation distances showed significant sensitivity to the grid even though the grid count on our medium grid is excessive by most standards. Further evaluation of the grid sensitivity is desired, but the calculations are becoming prohibitively expensive. Considering that we are only computing the solution over a pair of cylinders, the challenge of accurately simulating the solution over a full landing gear is evident. Nonetheless, our comparisons with experiment have been very encouraging. Good agreement has been obtained, especially for the larger separation distance. As noise source modeling is our ultimate goal, further validation of our computations using comparisons of the radiated noise are planned in subsequent work.

\section{Acknowledgment}

The authors would like to express their appreciation to Mr. M. R. Wiese of the Analytical Services and Materials, Inc. for generating the grid used during the computations.

\section{References}

1. Zdravkovich, M. M., "Review of Flow Interference Between Two Circular Cylinders in Various Arrangements,” J. Fluids Engineering, Vol., pp. 618-633, December 1977.

2. Ohya, Y., Okajima, A., and Hayashi, M., "Wake Interference and Vortex Shedding," Encyclopedia of Fluid Mechanics, edited by N.P. Cheremisinoff (Gulf Publishing Company, Houston), Vol. 8, pp.323$389,1989$.

3. Zdravkovich, M. M., "Flow Induced Oscillations of Two Interfering Circular Cylinders," J. of Sound and Vibrations, Vol. 101, No.4, pp. 511-521, 1985.

4. Igarashi, T., "Characteristics of the Flow around Two Circular Cylinders Arranged in Tandem $\left(1^{\text {st }}\right.$ Report)," Bulletin of the JSME, Vol.24, No. 188, pp. 323-331, February 1981. 
5. Igarashi, T., "Characteristics of the Flow around Two Circular Cylinders Arranged in Tandem (2 Report, Unique Phenomena at Small Spacing)," Bulletin of the JSME, Vol.27, No. 233, pp. 23802387, November 1984.

6. Ljungkrona, L., Norberg, CH., and Sunden, B., "Free-Stream Turbulence and Tube Spacing Effects on Surface Pressure Fluctuations for Two Tubes in an In-Line Arrangement," J. Fluid and Structures, Vol.5, pp.701-727, 1991.

7. Ljungkrona, L., and Sunden, B., "Flow Visualization and Surface Pressure Measurement on Two Tubes in an Inline Arrangement,” Experimental Thermal and Fluid Science, Vol.6, pp. 15-27, 1993.

8. Wu, J., Welch, L.W., Welsh, M.C., Sheriden, J., and Walker, G. J., "Spanwise Wake Structures of a Circular Cylinder and Two Circular Cylinders in Tandem," Experimental Thermal and Fluid Science, Vol.9, pp. 299-308, 1994.

9. Lin, J.-C., Yang, Y., and Rockwell, D., "Flow Past Two Cylinders in Tandem: Instantaneous and Averaged Flow Structure,” J. Fluid and Structures, Vol. 16, No. 8, pp. 1059-1071, December 2002.

10. Okajima, A., "Flows around Two Tandem Circular Cylinders at Very High Reynolds Numbers," Bulletin of the JSME, Vol.22, No. 166, pp. 504-511, April 1979.

11. Arie, M., Kiya, M., Moriya, M., and Mori, H., "Pressure Fluctuations on the Surface of Two Circular Cylinders in Tandem Arrangement,” J. Fluids Engineering, Vol. 105, pp. 161-167, June 1983.

12. Sun, T.F., Gu, Z.F., He, D.X., and Zhang, L. L., "Fluctuating Pressure on Two Circular Cylinders at High Reynolds Numbers,” J. Wind Engineering and Industrial Aerodynamics, Vol. 41-44, pp. 577588, 1992.

13. Gu, Z.F., Sun, T.F., He, D.X., and Zhang, L. L., "Two Circular Cylinders in High-Turbulence Flow at Supercritical Reynolds Number," J. Wind Engineering and Industrial Aerodynamics, Vol. 49, pp. 379-388, 1993.

14. Gu, Z. F., "On Interference Between two Circular Cylinders at Supercritical Reynolds Number,” J. Wind Engineering and Industrial Aerodynamics, Vol. 62, pp. 175-190, 1996.

15. Gu, Z. F., and Sun, T.F., "On Interference Between two Circular Cylinders in Staggered Arrangement at High Subcritical Reynolds Numbers," J. Wind Engineering and Industrial Aerodynamics, Vol. 80, pp. 287-309, 1999. 
16. Sun, J., Li, J., and Roux, B., "Flow Regimes and Frequency Selection of a Cylinder Oscillating in an Upstream Cylinder Wake," International J. for Numerical Methods in Fluids, Vol. 16, pp. 915-929, 1993.

17. Mittal, S., Kumar, V., and Raghuvanshi, A., "Unsteady Incompressible Flows Past two Cylinders in Tandem and Staggered Arrangements," International J. for Numerical Methods in Fluids, Vol. 25, pp. 1315-1344, 1997.

18. Itoh, Y., and Himeno, R., "Numerical Simulation of Three-Dimensional Flow Around two Circular Cylinders in Tandem Arrangement," RIKEN Review No. 48: Focused on High Performance Computing in RIKEN 2001, pp. 3-6, October 2002.

19. Jenkins, L. N., Khorrami, M. R., Choudhari, M. M., and McGinley, C. B., "Characterization of Unsteady Flow Structures around Tandem Cylinders for Component Interaction Studies in Airframe Noise," AIAA paper AIAA-2005-2812, May 2005.

20. Jenkins, L. N., Neuhart, D.H., McGinley, C. B., Choudhari, M. M., and Khorrami, M. R., "Measurements of Unsteady Wake Interference Between Tandem Cylinders," AIAA paper AIAA2006-3202, June 2006.

22. Khorrami, M.R., Choudhari, M.M., Jenkins, L.N., and McGinley, C.B., "Unsteady Flowfield Around Tandem Cylinders as Prototype for Component Interaction in Airframe Noise,” AIAA Paper 20052866, May 2005.

21. Hutcheson, F. V. and Brooks, T. F., "Noise Radiation fron Single and Multiple Rods Configurations," AIAA Paper 2006-2629, May 2006.

23. Menter, F., "Improved Two-Equation k- $\omega$ Turbulence Models for Aerodynamic Flows," NASA TM 103975, 1992.

24. Khorrami, M.R., Choudhari, M.M., Lockard, D.P., Jenkins, L.N., and McGinley, C.B., "Unsteady Flowfield Around Tandem Cylinders as Prototype for Component Interaction in Airframe Noise," Submitted for publication to AIAA J., 2006.

25. Rumsey, C. L., Sanetrik, M. D., Biedron, R. T., Melson, N. D., and Parlette, E. B., "Efficiency and Accuracy of Time-Accurate Turbulent Navier-Stokes Computations," Computers \& Fluids, Vol. 25, No. 2, pp. 217-236, 1996. 
26. Choudhari, M. M. and Khorrami, M. R., "Slat Cove Unsteadiness: Effect of 3D Flow Structures," AIAA Paper 2006-0211, 2006.

27. Bendat, J. and Piersol, A., "Engineering Applications of Correlations and Spectral Analysis, $2^{\text {nd }}$ Ed.,” John Wiley \& Sons, New York, p. 49, 1993. 

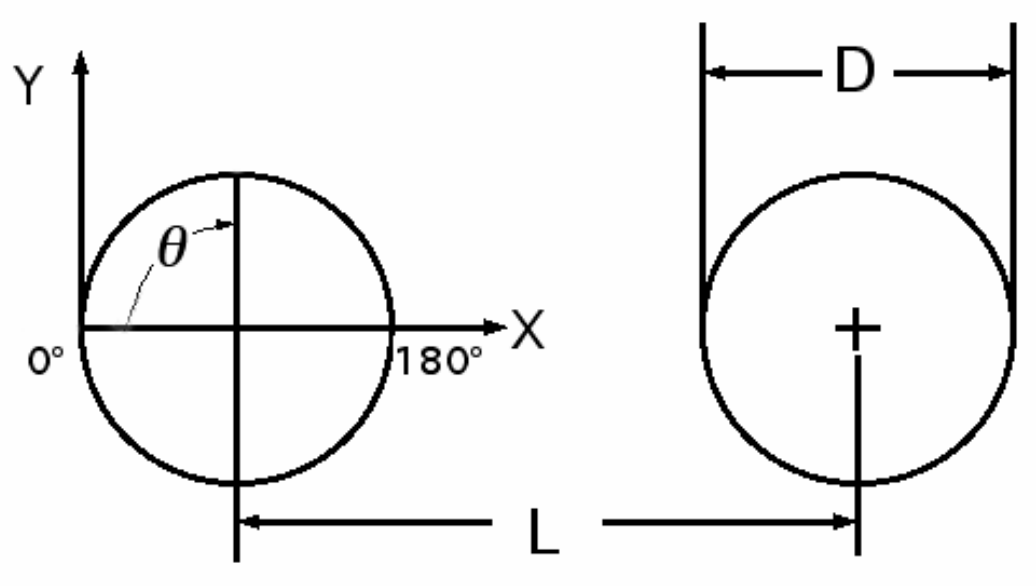

Fig.1. Schematic diagram of simulated configuration

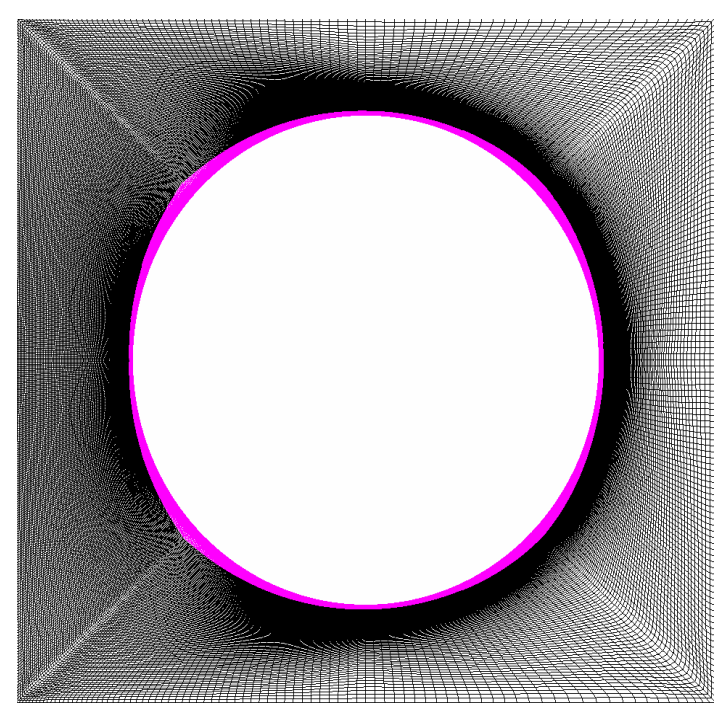

Fig. 2. Fully turbulent flow zone highlighted by purple color

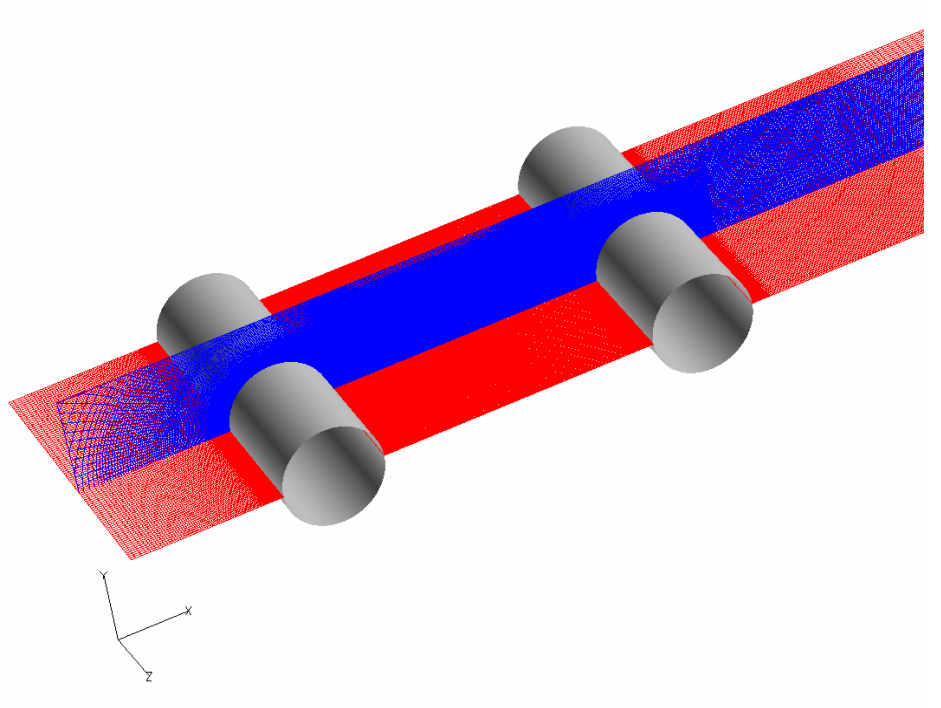

Fig. 3. Perspective view of 3-D fine grid for $L / D=3.7$ 


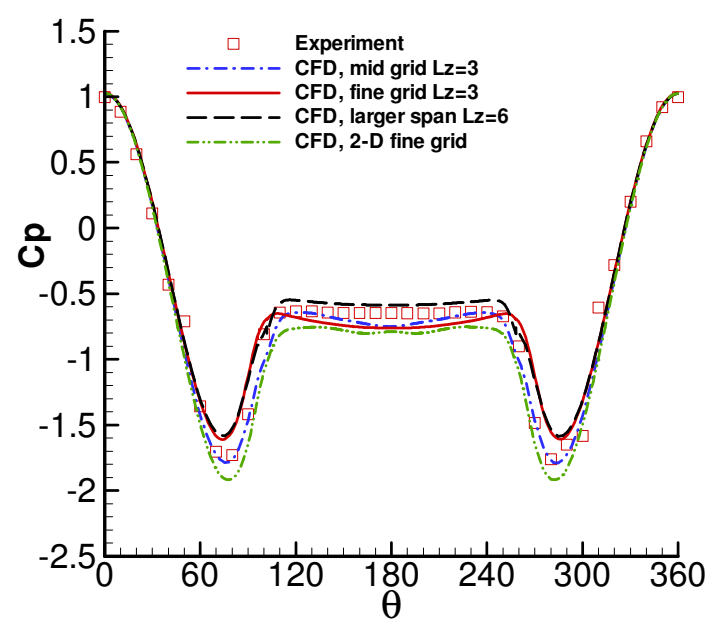

a) Front cylinder

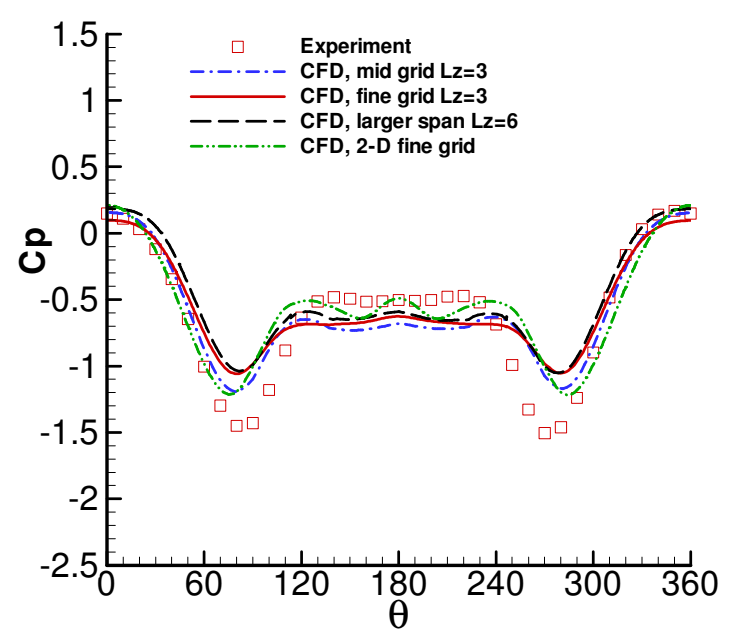

b) Rear cylinder

Fig. 4. Surface pressure distribution for $\mathrm{L} / \mathrm{D}=3.7$

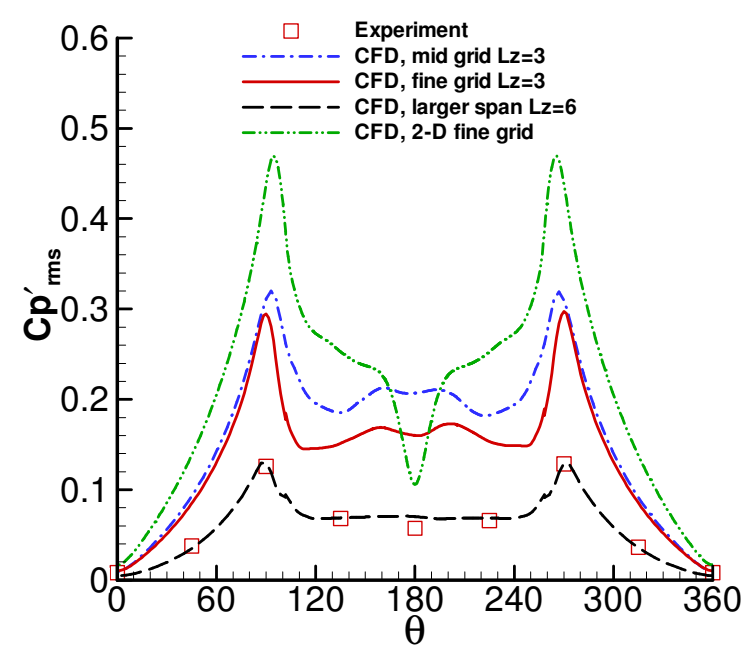

a) Front cylinder

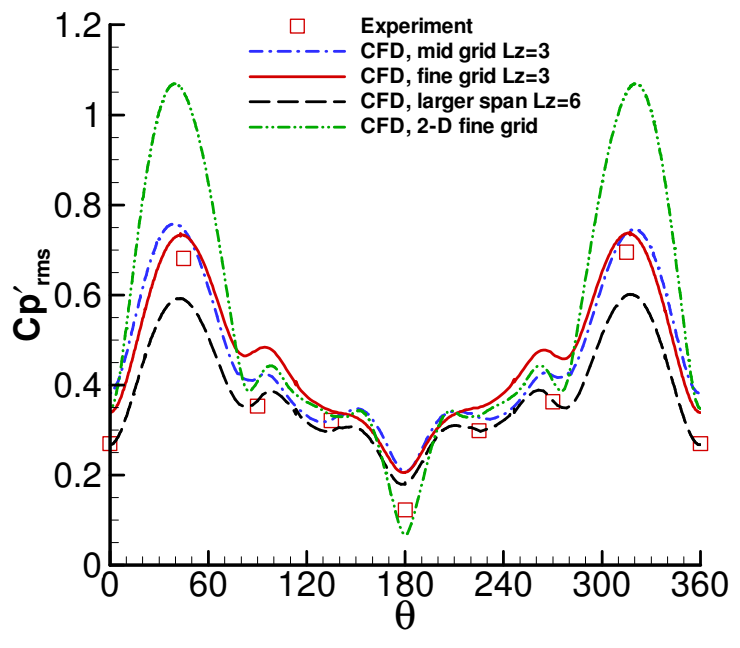

b) Rear cylinder

Fig. 5. Surface rms pressure distribution for $\mathrm{L} / \mathrm{D}=3.7$ 


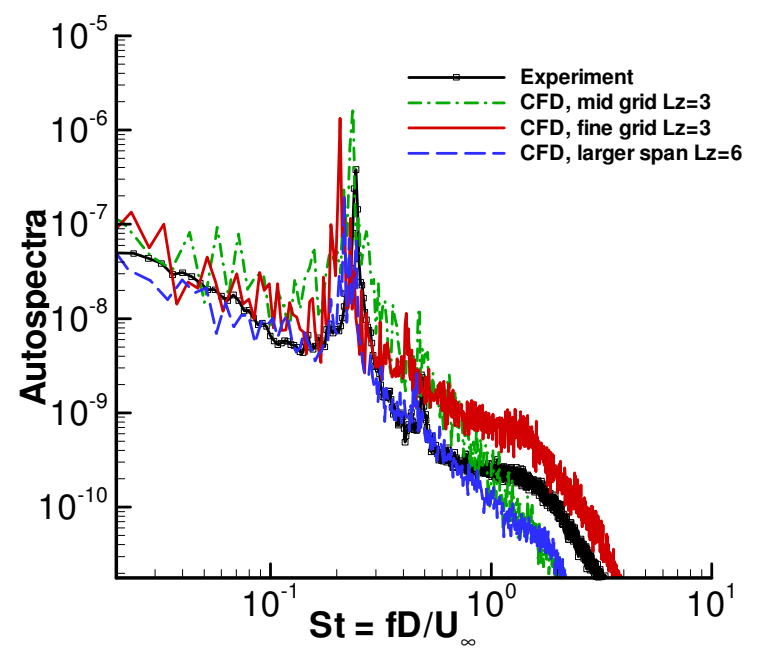

a) Front cylinder

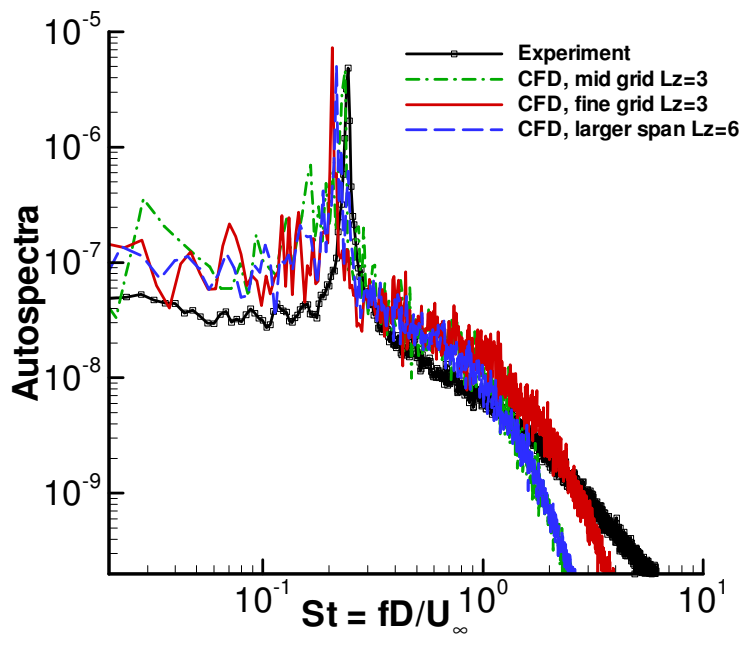

b) Rear cylinder

Fig. 6. Surface pressure spectrum at $\theta=135$ degrees for $L / D=3.7$ 


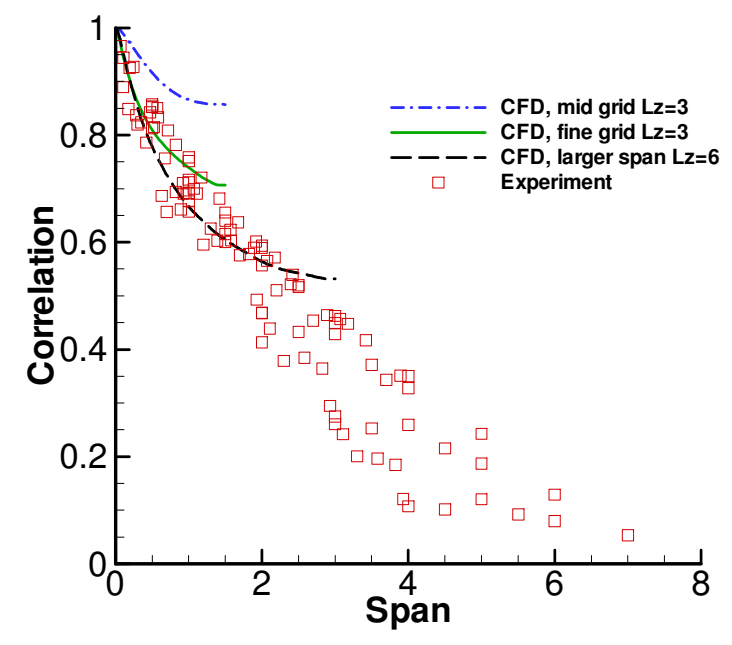

a) Front cylinder

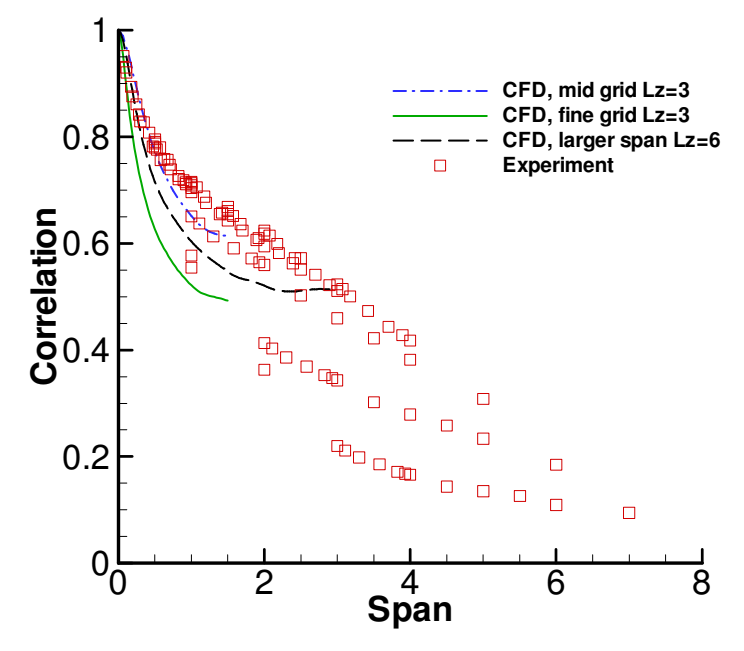

b) Rear cylinder

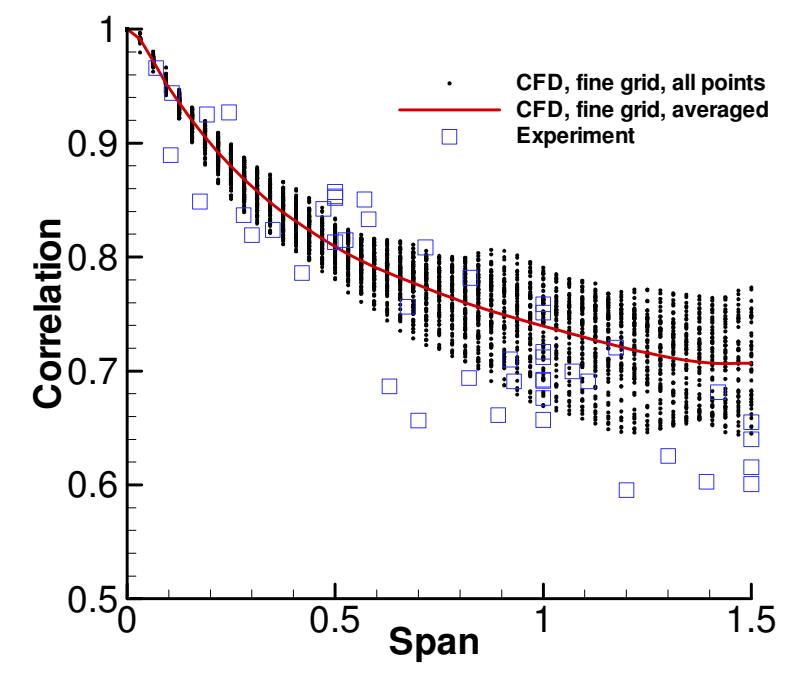

c) Computed scatter on front cylinder

Fig. 7. Surface pressure spanwise correlation at $\theta=135$ degrees for $L / D=3.7$ 


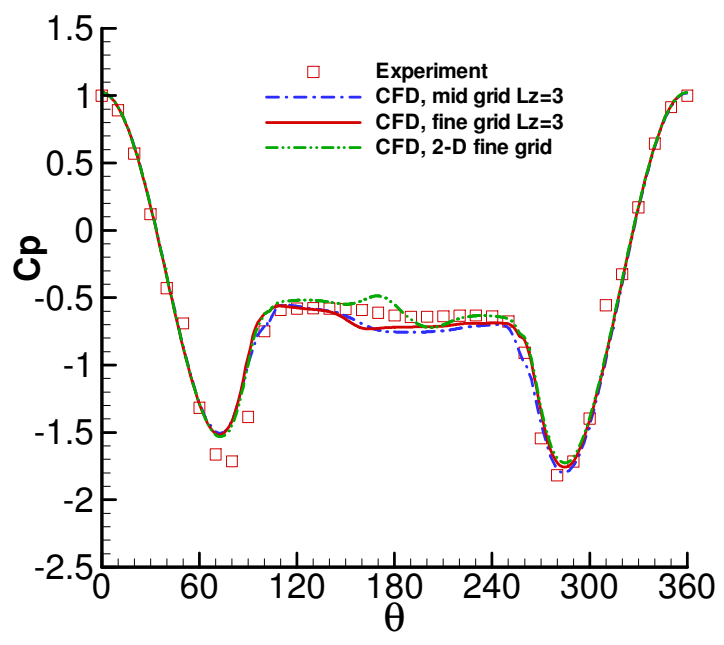

a) Front cylinder

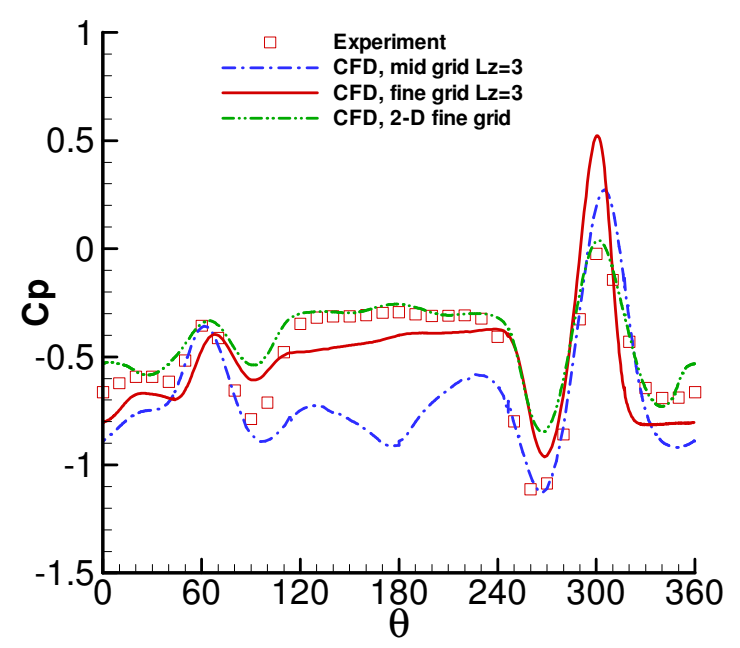

b) Rear cylinder

Fig. 8. Surface pressure distribution for $\mathrm{L} / \mathrm{D}=1.435$

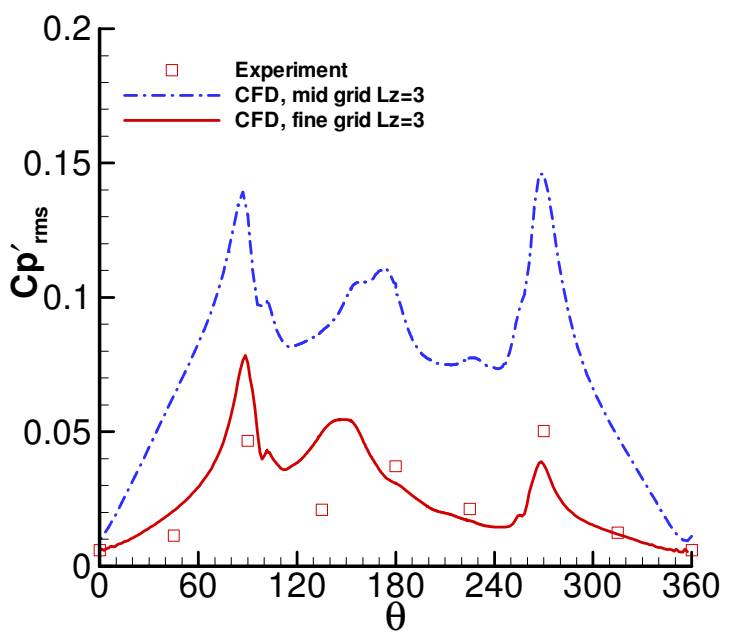

a) Front cylinder

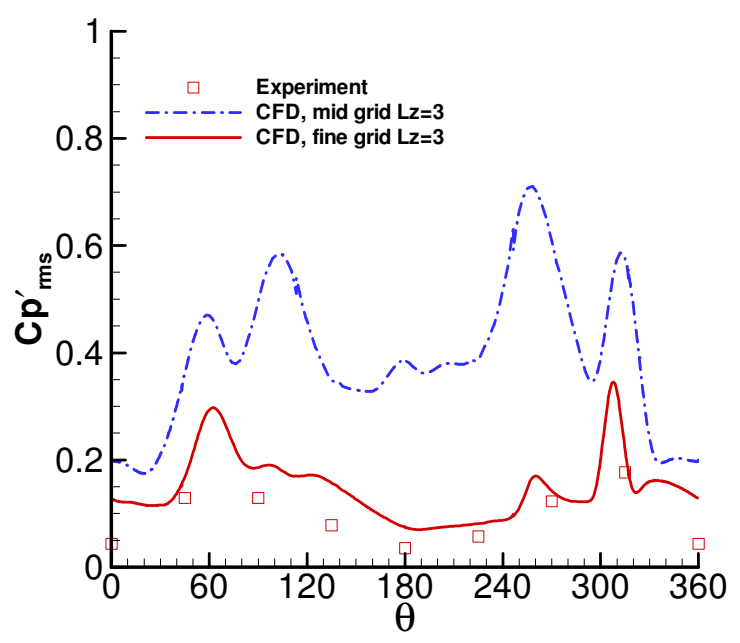

b) Rear cylinder

Fig. 9. Surface rms pressure distribution for $L / D=1.435$ 


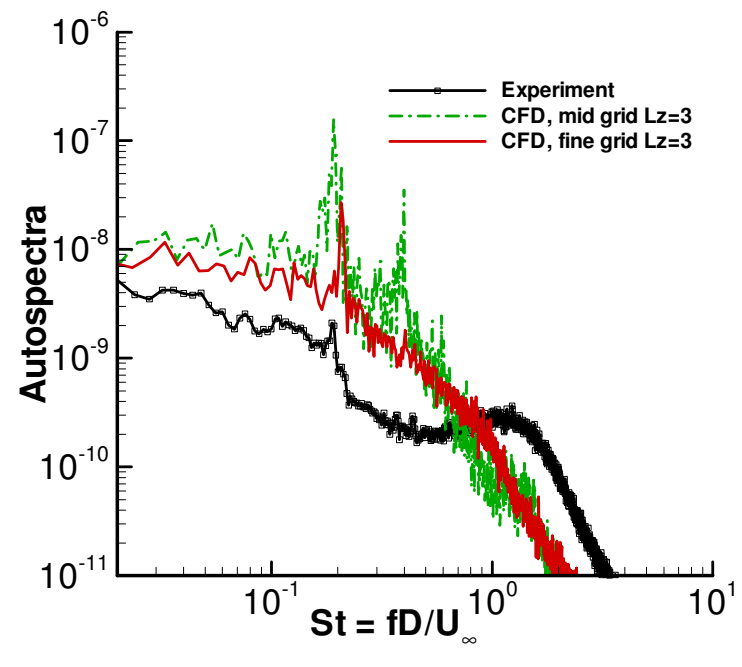

a) Front cylinder

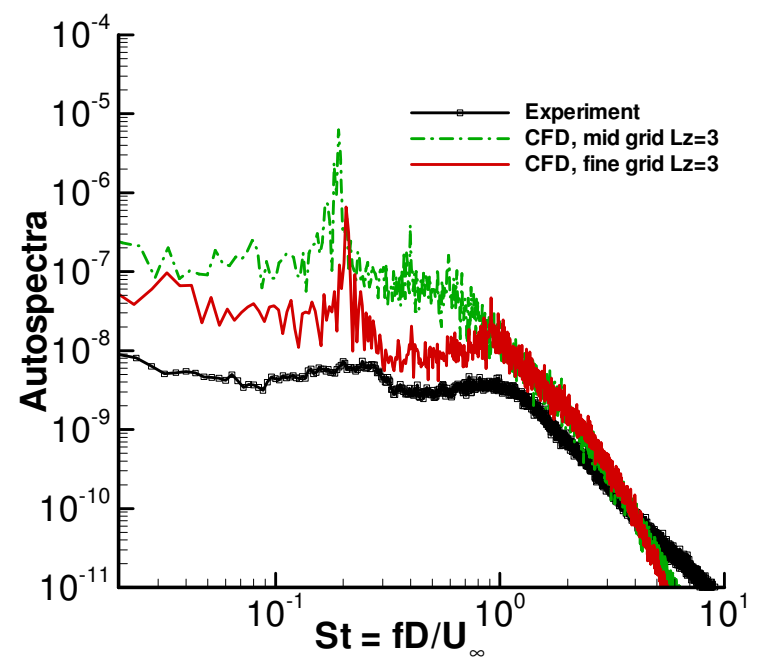

b) Rear cylinder

Fig. 10. Surface pressure spectrum at $\theta=135$ degrees for $\mathrm{L} / \mathrm{D}=1.435$

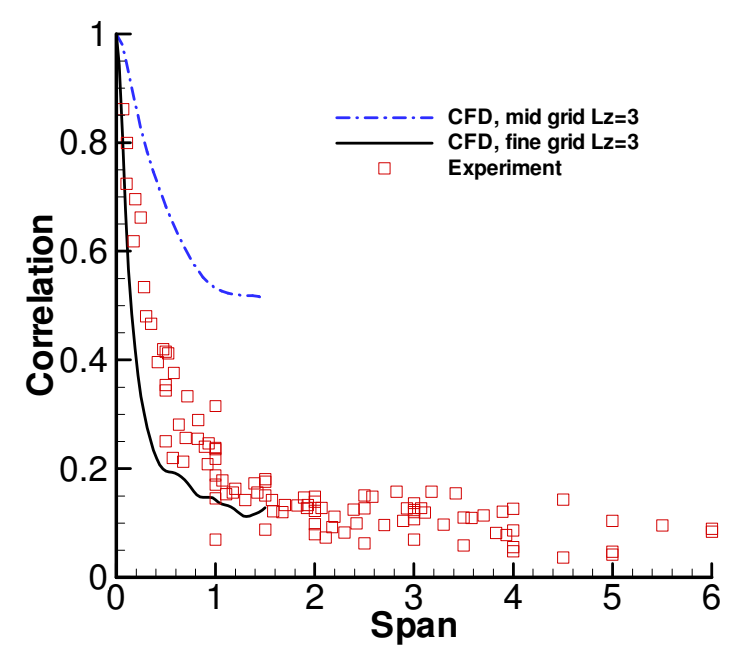

a) Front cylinder

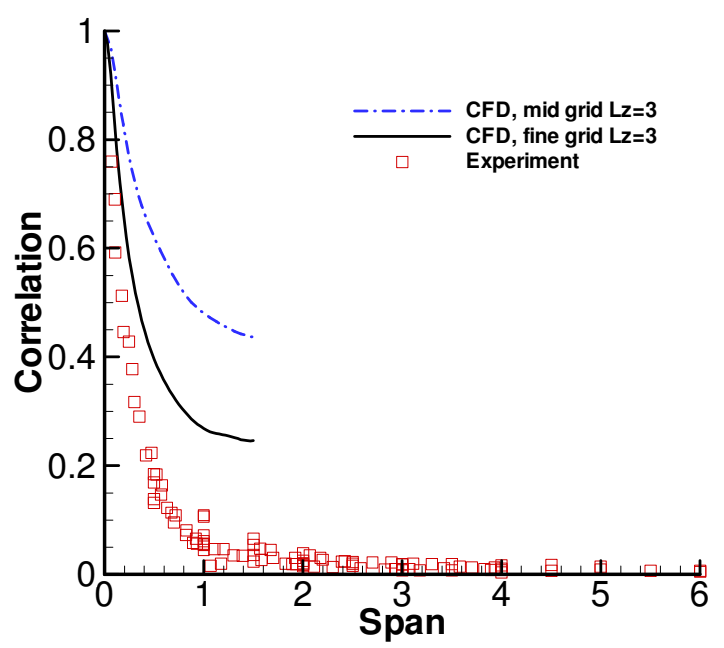

b) Rear cylinder

Fig. 11. Surface pressure spanwise correlation at $\theta=135$ degrees for $\mathrm{L} / \mathrm{D}=1.435$ 
Computation

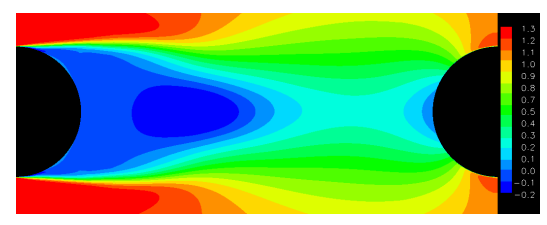

Fig. 12a. Streamwise velocity

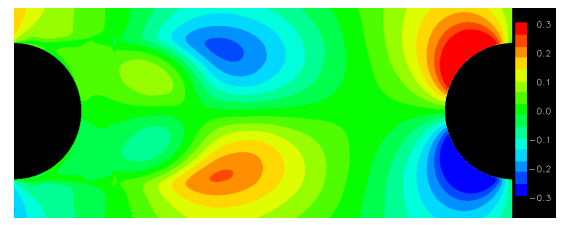

Fig. 12c. Vertical velocity

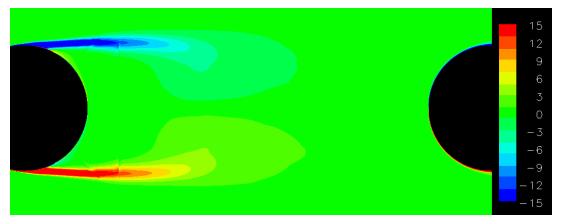

Fig. 12e. Spanwise vorticity
Experiment

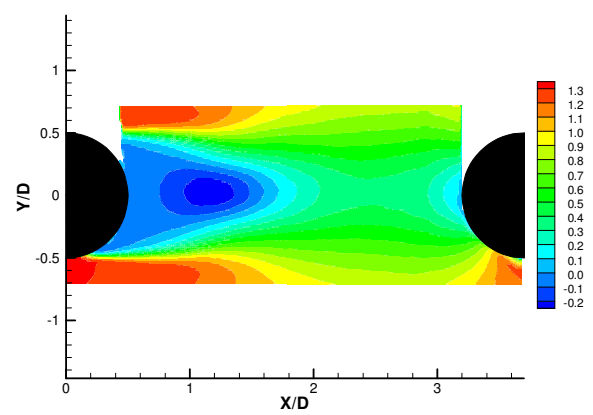

Fig. 12b. Streamwise velocity

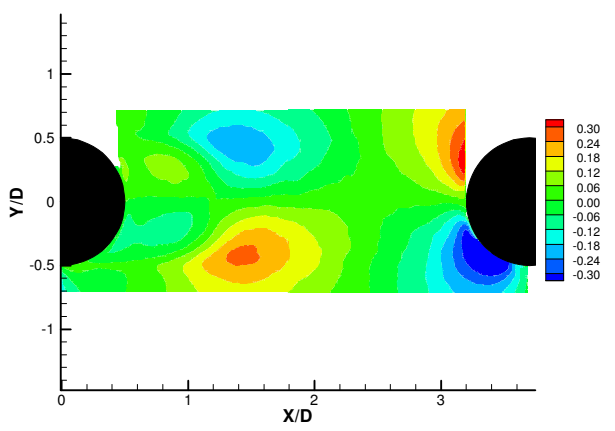

Fig. 12d. Vertical velocity

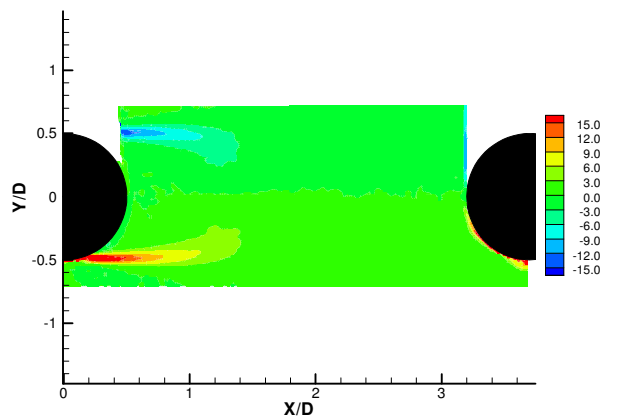

Fig. 12f. Spanwise vorticity

Fig. 12. Mean velocity and vorticity fields for $L / D=3.7$ 
Computation

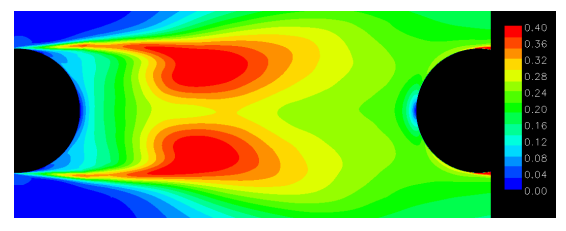

Fig. 13a. Streamwise velocity

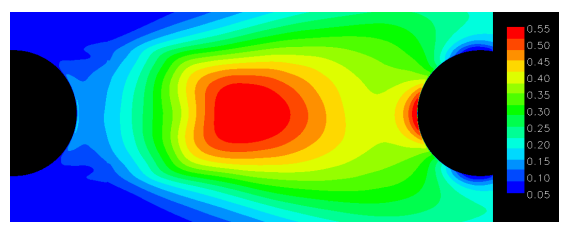

Fig. 13c. Vertical velocity

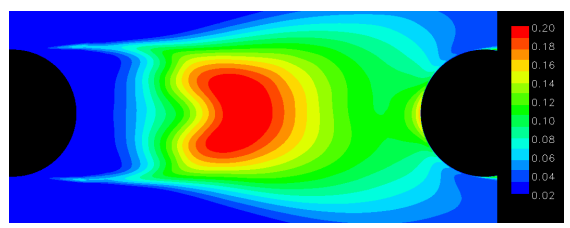

Fig. 13e. TKE
Experiment

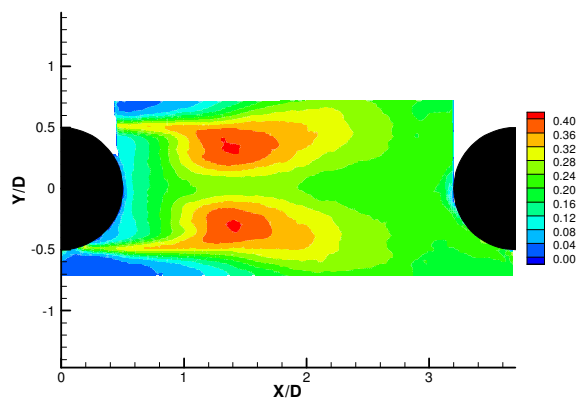

Fig. 13b. Streamwise intensities

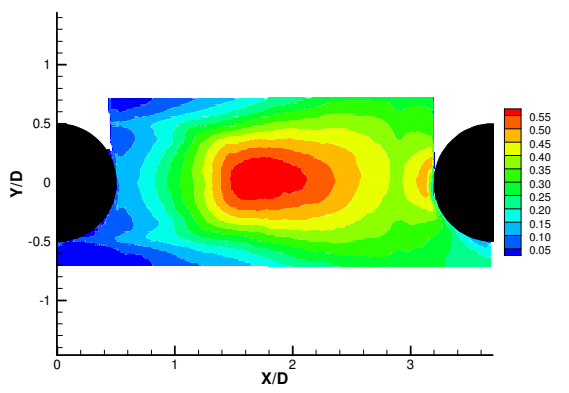

Fig. 13d. Vertical intensities

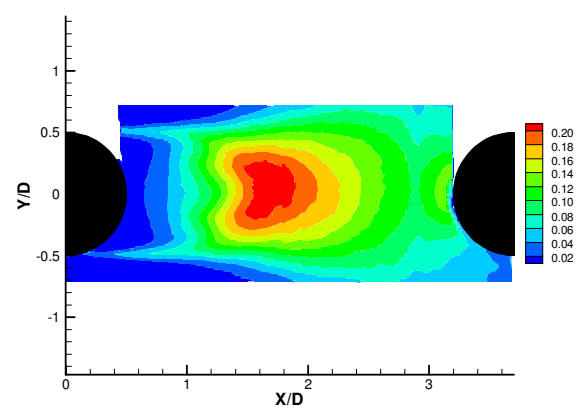

Fig. 13f. TKE

Fig. 13. Fluctuating velocity field for $\mathrm{L} / \mathrm{D}=3.7$ 
Computation

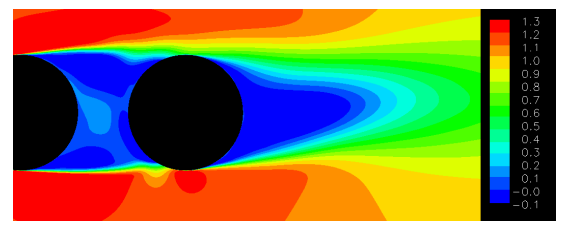

Fig. 14a. Streamwise velocity

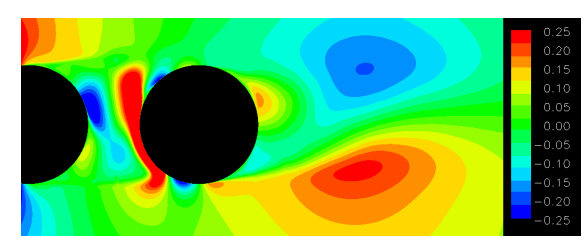

Fig. 14c. Vertical velocity

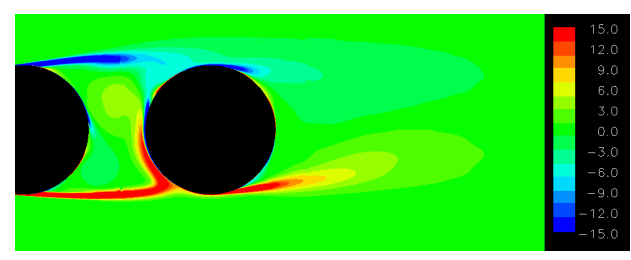

Fig. 14e. Spanwise vorticity

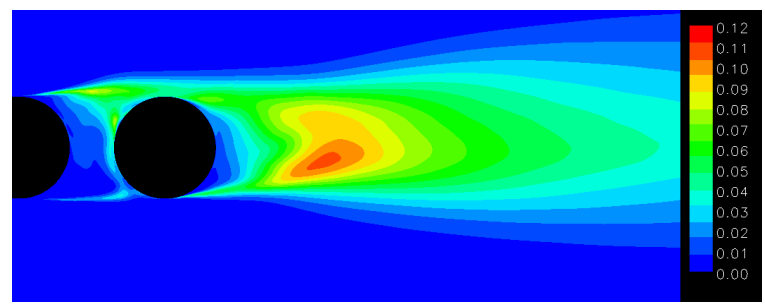

Fig. 14g. TKE
Experiment

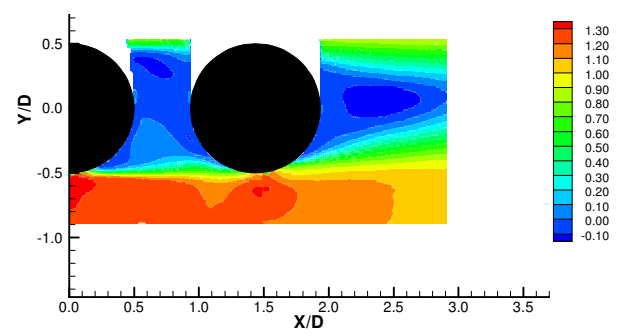

Fig. 14b. Streamwise velocity

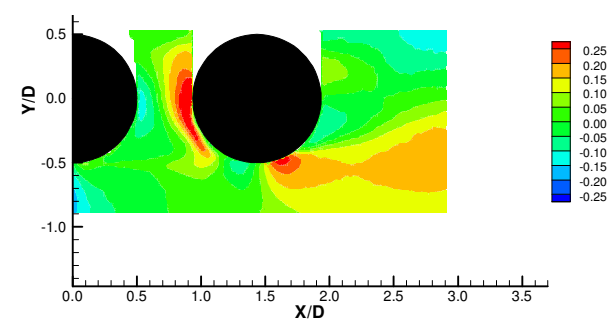

Fig. 14d. Vertical velocity

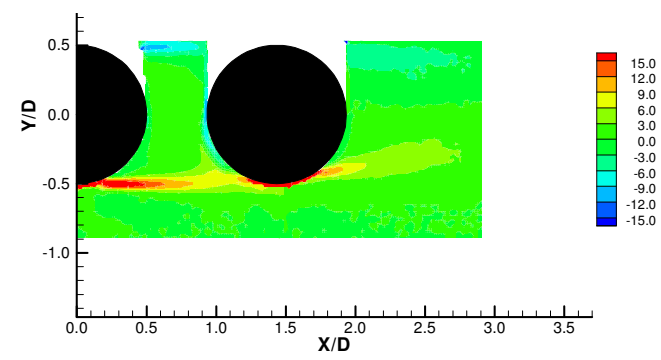

Fig. 14f. Spanwise vorticity

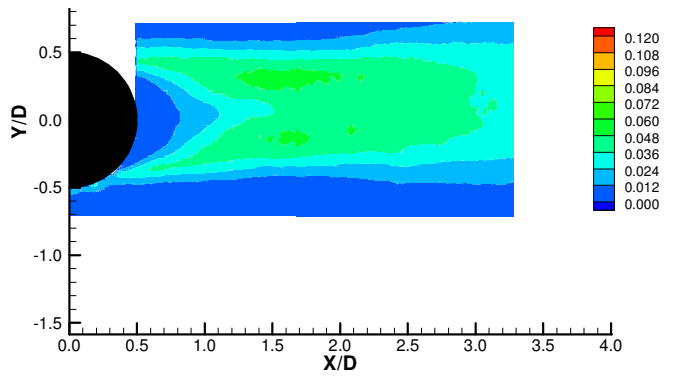

Fig. 14h. TKE

Fig. 14. Mean flow and TKE fields for $\mathrm{L} / \mathrm{D}=1.435$ 


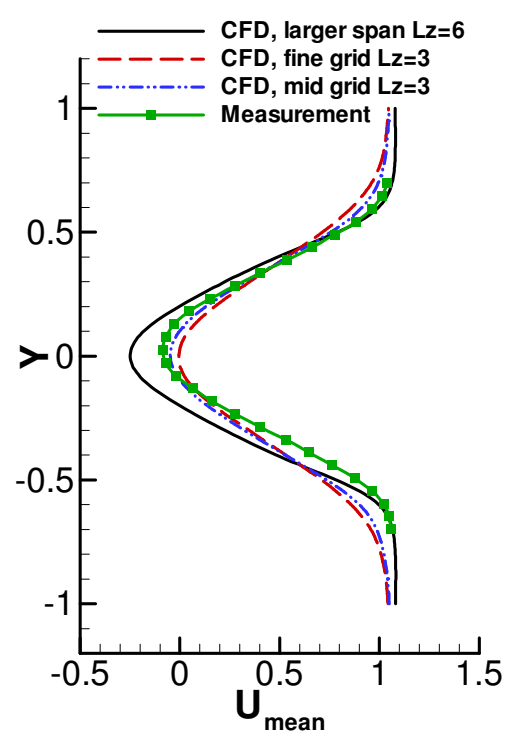

Fig. 15a. Mean streamwise velocity

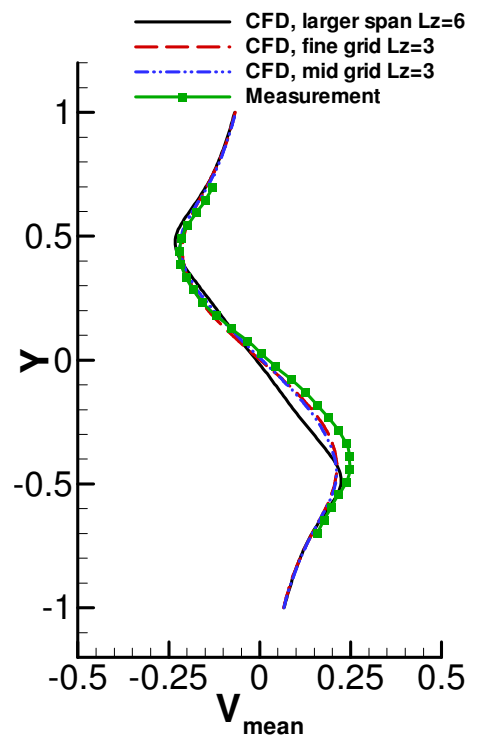

Fig. 15b. Mean vertical velocity

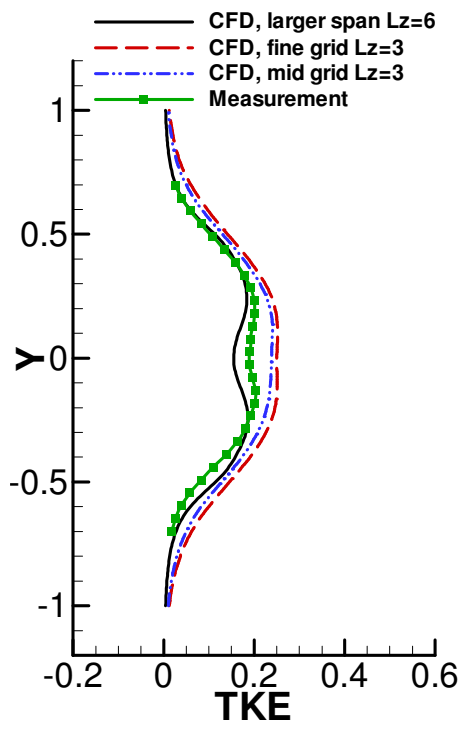

Fig. 15c. TKE

Fig. 15. Averaged flow profiles $1.5 \mathrm{D}$ downstream of the center of the front cylinder for $\mathrm{L} / \mathrm{D}=3.7$

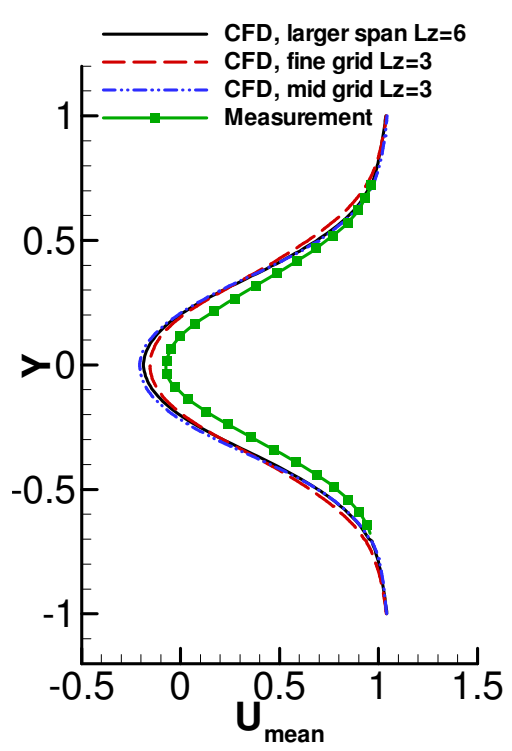

Fig. 16a. Mean streamwise

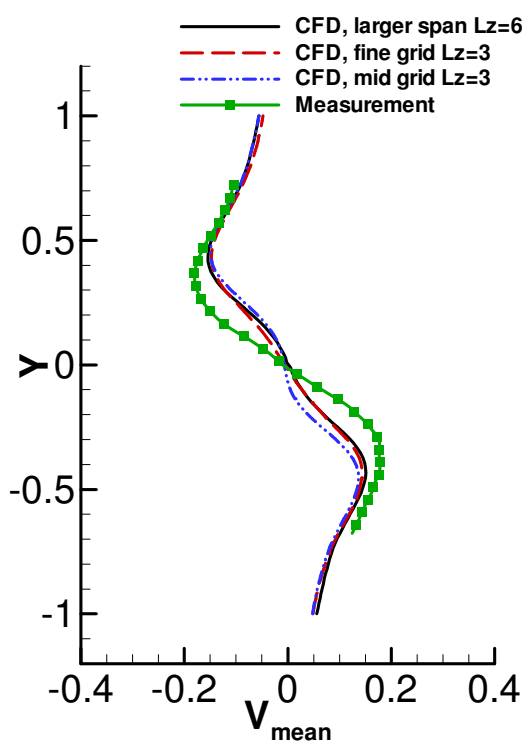

Fig. 16b. Mean vertical velocity

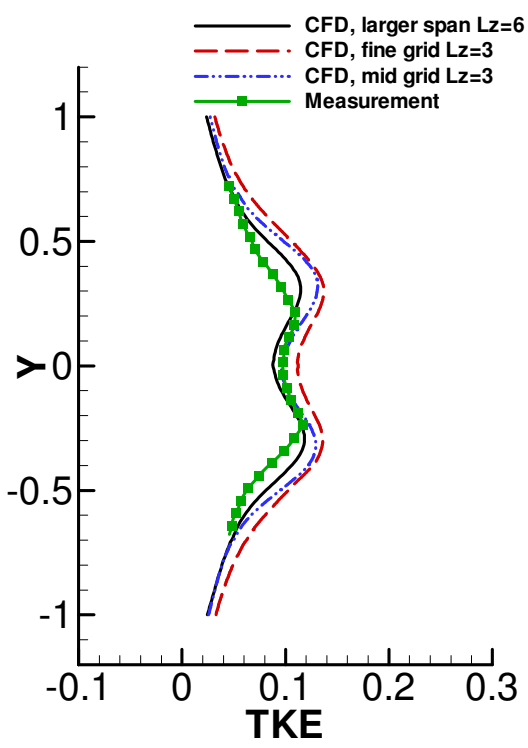

Fig. 16c. TKE velocity

Fig. 16. Averaged flow profiles $0.75 \mathrm{D}$ downstream of the center of the rear cylinderfor $\mathrm{L} / \mathrm{D}=3.7$ 


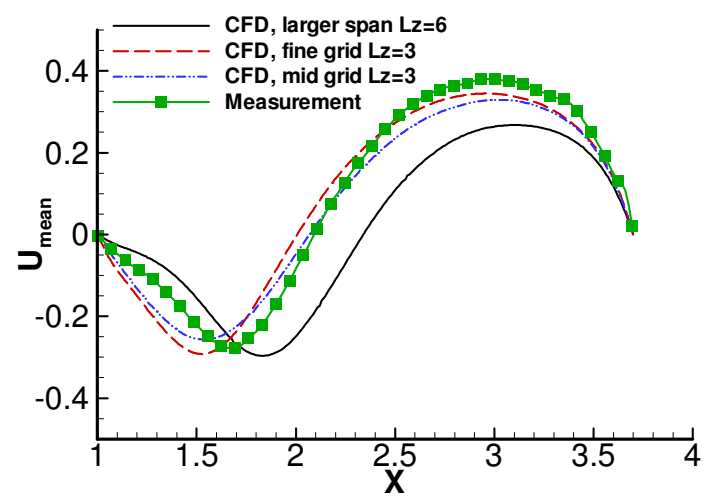

Fig. 17a. Mean streamwise velocity

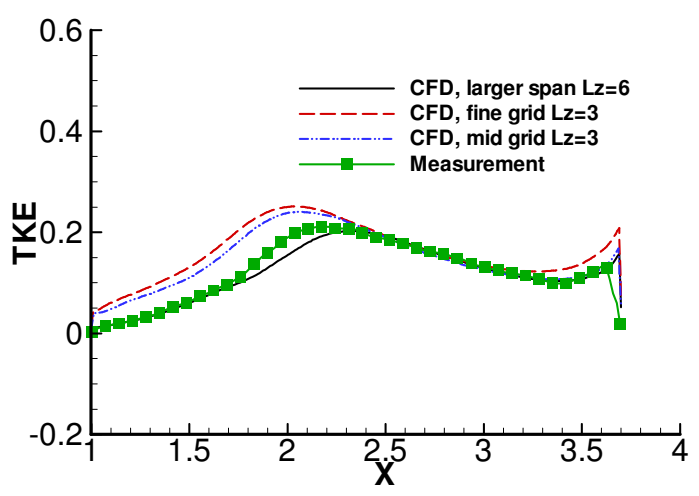

Fig. 17b. TKE

Fig. 17. Averaged flow profiles in between two cylinders along $y=0$ for $L / D=3.7$

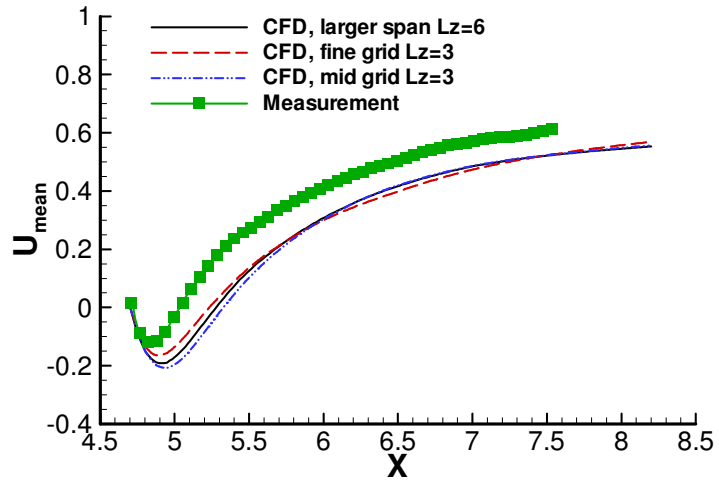

Fig. 18a. Mean streamwise velocity

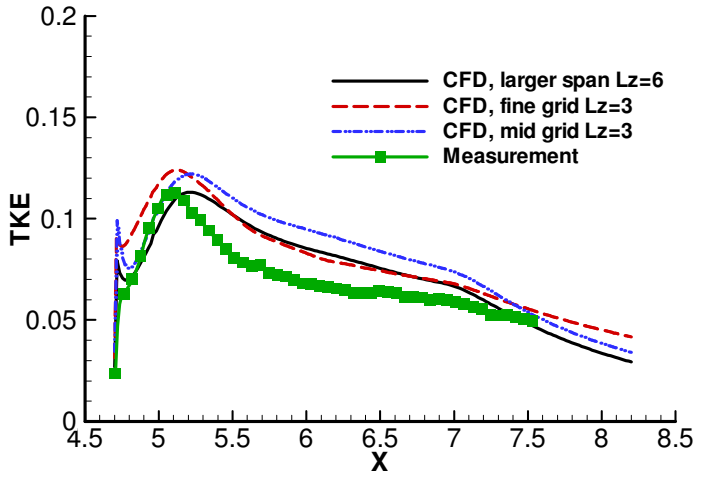

Fig. 18b. TKE

Fig. 18. Averaged flow profiles downstream of rear cylinder along $y=0$ for $L / D=3.7$ 


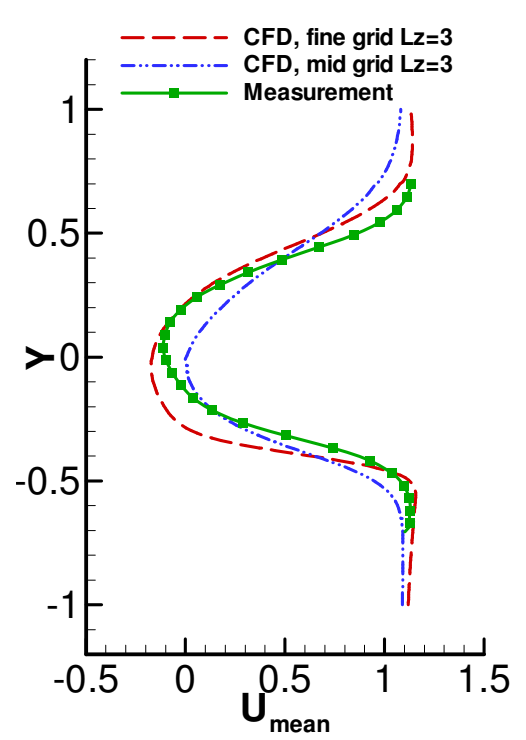

Fig. 19a. Mean streamwise velocity

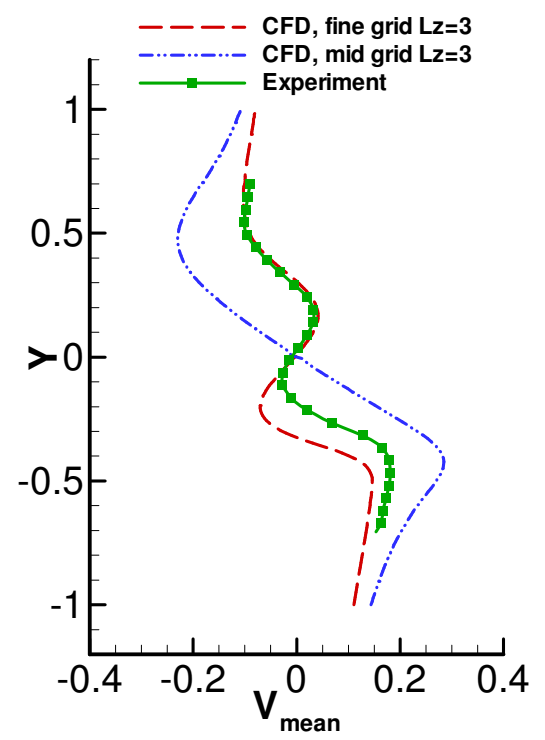

Fig. 19b. Mean vertical velocity

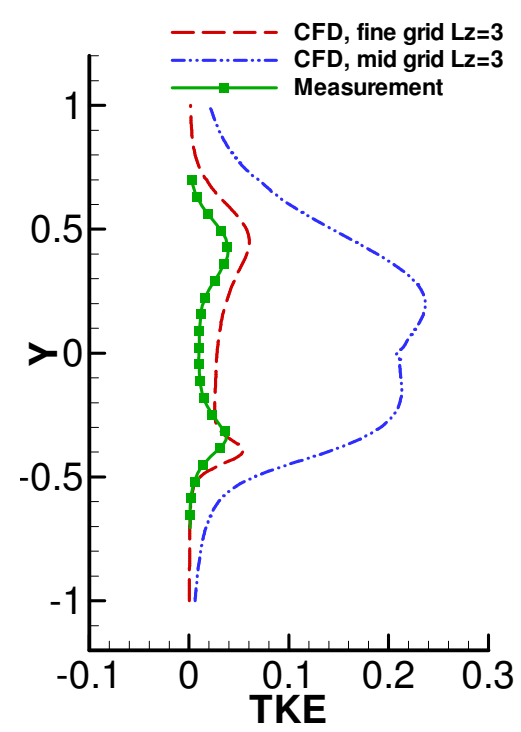

Fig. 19c. TKE

Fig. 19. Averaged flow profiles $0.75 \mathrm{D}$ downstream of rear cylinder centerline for $\mathrm{L} / \mathrm{D}=1.435$

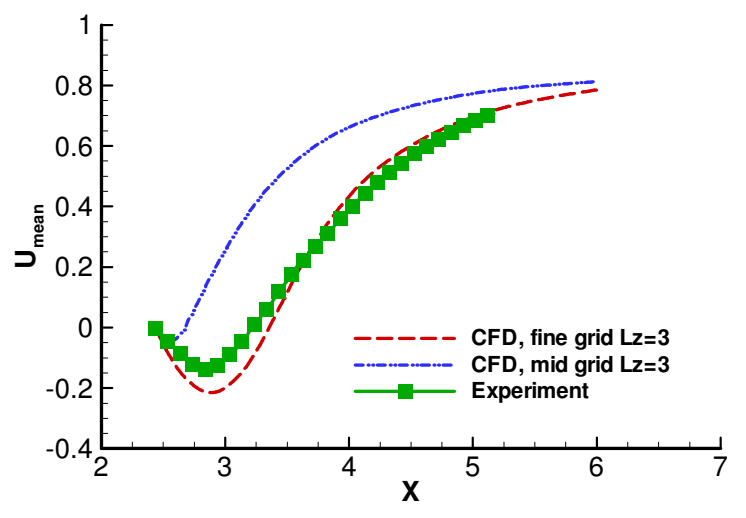

Fig. 20a. Mean streamwise velocity

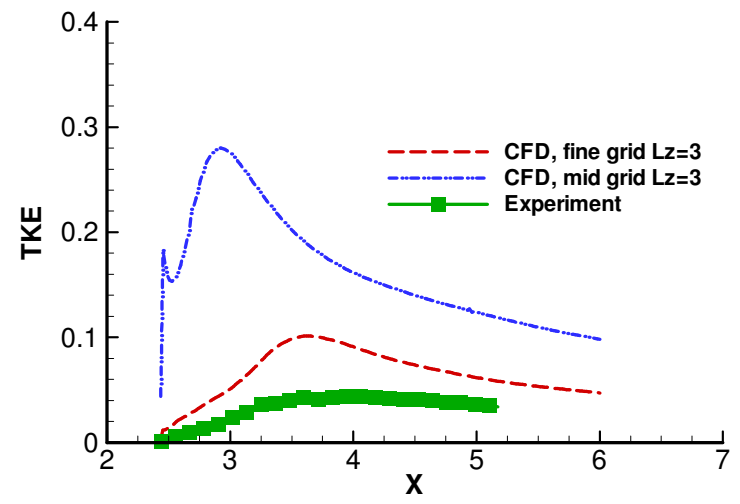

Fig. 20b. TKE

Fig. 20. Averaged flow profiles downstream of rear cylinder along $y=0$ for $L / D=1.435$ 

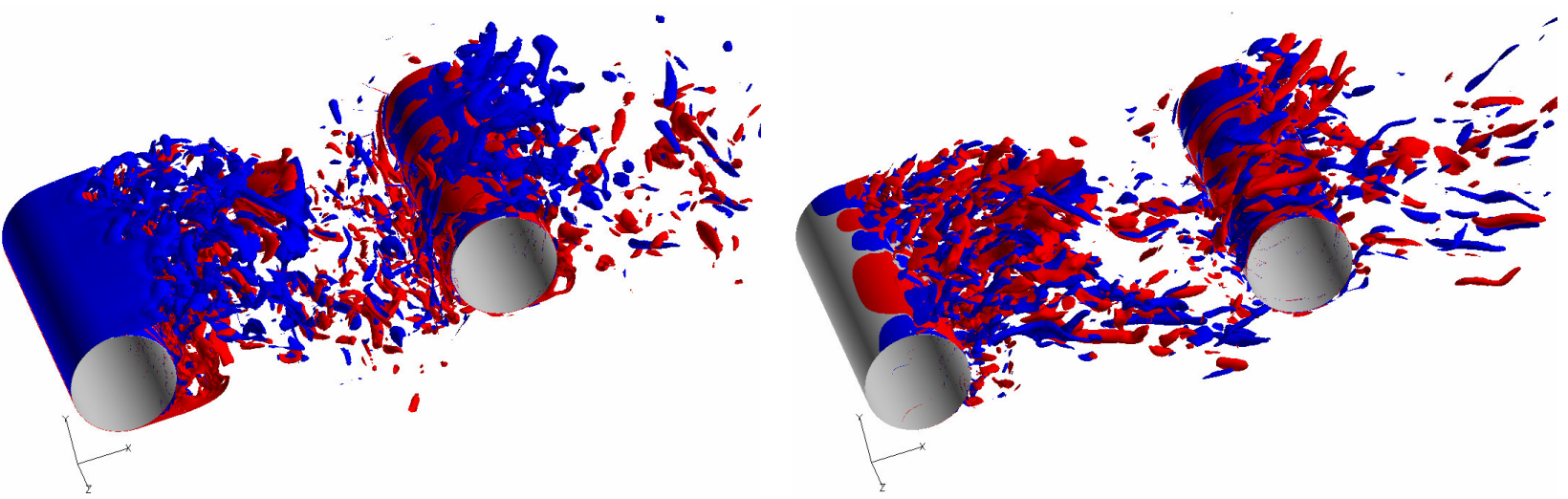

Fig. 21a. Spanwise component. Magnitude is 12 Fig. 21b. Streamwise component. Magnitude is 12

Fig. 21. Iso-surface of vorticity field for $\mathrm{L} / \mathrm{D}=3.7$. Counterclockwise vorticity is red, and clockwise is blue.

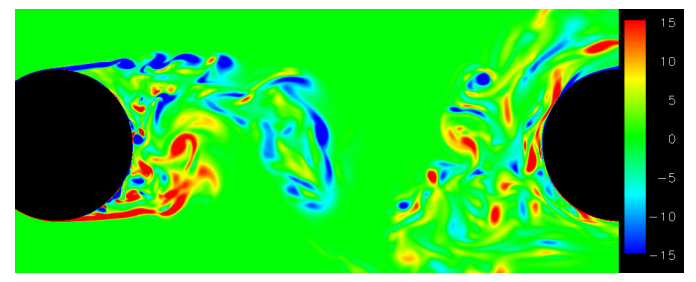

Fig. 22a. Computation

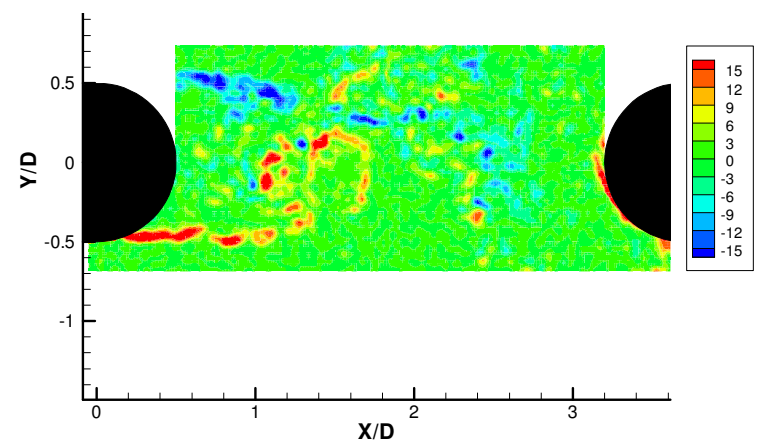

Fig. 22b. Experiment

Fig. 22. Instantaneous spanwise vorticity for $\mathrm{L} / \mathrm{D}=3.7$ 


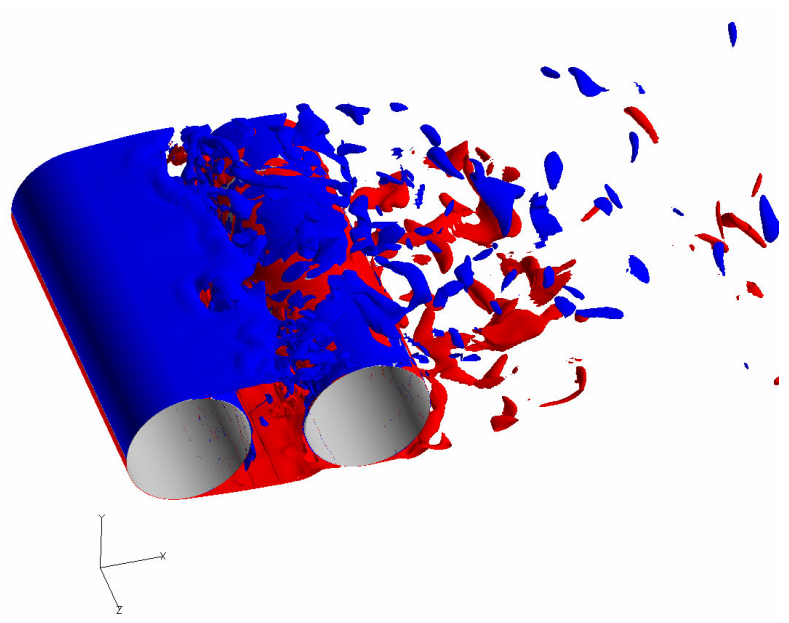

Fig. 23a. Spanwise component. Magnitude is 12

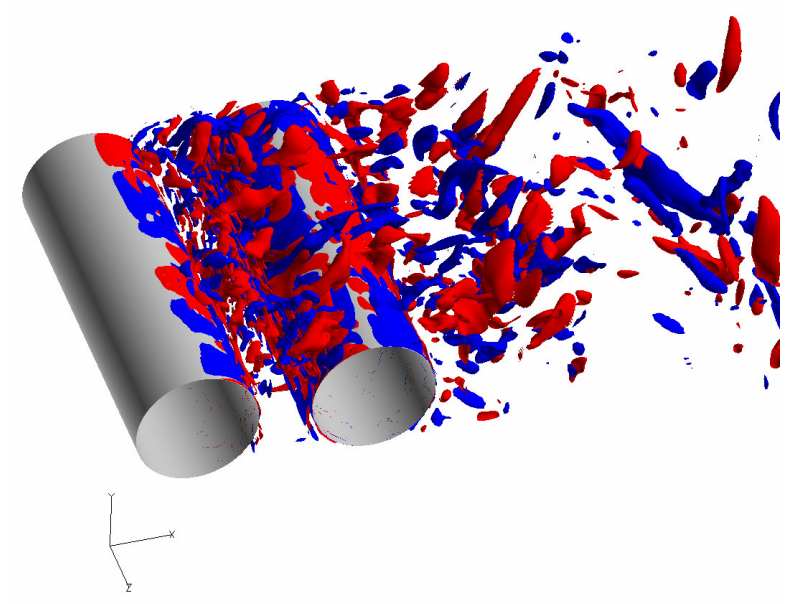

Fig. 23b. Vertical component. Magnitude is 6

Fig. 23. Iso-surface of vorticity field for $\mathrm{L} / \mathrm{D}=1.435$. Counterclockwise vorticity is red, and clockwise is blue. 